\title{
CRITICAL POINTS OF AMBROSIO-TORTORELLI CONVERGE TO CRITICAL POINTS OF MUMFORD-SHAH IN THE ONE-DIMENSIONAL DIRICHLET CASE
}

\author{
Gilles A. Francfort ${ }^{1}$, Nam Q. LE ${ }^{2}$ and Sylvia Serfaty ${ }^{3}$
}

\begin{abstract}
Critical points of a variant of the Ambrosio-Tortorelli functional, for which non-zero Dirichlet boundary conditions replace the fidelity term, are investigated. They are shown to converge to particular critical points of the corresponding variant of the Mumford-Shah functional; those exhibit many symmetries. That Dirichlet variant is the natural functional when addressing a problem of brittle fracture in an elastic material.
\end{abstract}

Mathematics Subject Classification. 49Q20, 49J45, 35B38, 35J60.

Received September 19, 2007. Revised February 29, 2008.

Published online June 24, 2008.

\section{INTRODUCTION}

In the late 80 's, Mumford and Shah proposed a new functional for image segmentation in their celebrated paper [13]. If $g \in L^{\infty}(\Omega ;[0,1])$ represents a continuous interpolation of the collected pixelated data over the image domain $\Omega \subset \mathbb{R}^{2}$, then the proposed segmentation consists in minimizing

$$
(u, K) \mapsto \mathcal{M S}(u, K):=\int_{\Omega \backslash K}|\nabla u|^{2} \mathrm{~d} x+2 \mathcal{H}^{1}(K)+\lambda \int_{\Omega}(u-g)^{2} \mathrm{~d} x
$$

among all compact subsets $K \subset \Omega$ and all $u \in H^{1}(\Omega \backslash K)$. In that functional, $\lambda$ is a positive weight left to the investigator's appreciation, $K$ represents the contours of the image, and $u$ the resulting grey contrast $(0 \leq u(x) \leq 1)$

Proving existence for minimizers of that functional was not a trivial task and it gave rise to a abundant literature spearheaded by the work of De Giorgi and that of Ambrosio on the space $S B V(\Omega)$; see e.g. [1].

\footnotetext{
Keywords and phrases. Mumford-Shah functional, Ambrosio-Tortorelli functional, Gamma-convergence, critical points, brittle fracture.

${ }^{1}$ LPMTM, Université Paris 13, Av. J.B. Clément, 93430 Villetaneuse, France. francfor@galilee.univ-paris13.fr Partially supported by ANR-06-BLAN-0082.

2 Courant Institute of Mathematical Sciences, 251 Mercer St, New York, NY 10012, USA. quangle@cims.nyu.edu

Partially supported by a Vietnam Education Foundation graduate Fellowship.

3 Courant Institute of Mathematical Sciences, 251 Mercer St, New York, NY 10012, USA. serfaty@cims.nyu.edu

Supported by NSF CAREER grant \#DMS0239121 and a Sloan Foundation Fellowship.
} 
The underlying idea was to view $\mathcal{M} S(u, K)$ as a one field functional

$$
M S(u)=\int_{\Omega}|\nabla u|^{2} \mathrm{~d} x+2 \mathcal{H}^{1}(S(u))+\lambda \int_{\Omega}(u-g)^{2} \mathrm{~d} x,
$$

over $S B V(\Omega)$, the space of functions $u \in L^{1}(\Omega)$ such that their distributional derivative is a Radon measure $D u$ with finite total variation $|D u|(\Omega)$, jump set $S(u)$ (the complement of the set of Lebesgue points for $u$ ), and no Cantor part. The next step was to prove existence of a minimizer $u_{m}$ of $M S$ in that space, and then to show that the pair $\left(u_{m}, \overline{S\left(u_{m}\right)}\right)$ was actually a minimizer for $\mathcal{M S}$. That program was successfully completed, culminating in [8].

From a computational standpoint, the search for a minimizer of (1.1) is not easy, because the test fields exhibit discontinuities at unknown locations and the implementation of classical finite element methods becomes a perilous endeavor. A possible remedy consists in resorting to variational convergence, specifically $\Gamma$-convergence, so as to approximate $M S$ by a more regular functional - denoted henceforth by $A T_{\varepsilon}$ - whose minimizers are easier to evaluate. For more information on $\Gamma$-convergence, we refer the interested reader to e.g. [7] and merely emphasize for now that an important property of $\Gamma$-convergence is that (approximate) minimizers of $A T_{\varepsilon}$ that converge as $\varepsilon \searrow 0$ will converge to bona fide minimizers of $M S$.

There is by now an abundant literature on the approximation of the Mumford-Shah functional and many approximating sequences have been proposed. The most computationally efficient in our opinion is that originally proposed by Ambrosio and Tortorelli in [2,3], in the footstep of the functional proposed by Modica and Mortola for the approximation of the perimeter [12]. Consider

$$
A T_{\varepsilon}(u, v)=\int_{\Omega}\left(\left(\eta_{\varepsilon}+v^{2}\right)|\nabla u|^{2}+\varepsilon|\nabla v|^{2}+\frac{(1-v)^{2}}{\varepsilon}\right) \mathrm{d} x+\lambda \int_{\Omega}(u-g)^{2} \mathrm{~d} x,
$$

with $0<\eta_{\varepsilon} \ll \varepsilon$. It is proved in $[2,3]$ that $A T_{\varepsilon} \Gamma(\mathcal{B}(\Omega) \times \mathcal{B}(\Omega))$-converges to $M S$, suitably extended to a two-field functional as

$$
M S(u, v)=\left\{\begin{array}{cl}
M S(u) & \text { if } v \equiv 1 \\
+\infty & \text { otherwise. }
\end{array}\right.
$$

Above, $\mathcal{B}(\Omega)$ stands for the set of all Borel functions on $\Omega$, and the convergence is the convergence in measure. Actually, we can also view the convergence as taking place in $L^{2}(\Omega) \times L^{2}(\Omega)$.

The functional $A T_{\varepsilon}$ is easily seen, through the direct method of the Calculus of Variations, to admit at least one minimizing pair $\left(u_{\varepsilon}, v_{\varepsilon}\right) \in H^{1}(\Omega) \times H^{1}(\Omega)$, for any fixed value of $\varepsilon$. The associated sequence is bounded in e.g. $L^{\infty}(\Omega) \times L^{\infty}(\Omega)$, and a subsequence can be shown to converge in measure (and also strongly in $\left.L^{2}(\Omega) \times L^{2}(\Omega)\right)$ to $(u, v \equiv 1)$, which, by the already evoked property of $\Gamma$-convergence, will be a minimizer for $M S$.

In an apparently disconnected context, recent years have witnessed the birth of a variational theory of brittle fracture evolution. One of its constitutive elements is that, at each time, the total energy of the system, the sum of the elastic and surface energies, is to be minimized among all admissible competitors [10]. That total energy is a close parent of the Mumford-Shah functional $M S$ for image segmentation. It is given - say in antiplane shear, for which the displacement field is unidirectional, and for normalized shear modulus and fracture toughness - by

$$
\mathcal{F}(u, v)=\left\{\begin{array}{cl}
\int_{\Omega}|\nabla u|^{2} \mathrm{~d} x+2 \mathcal{H}^{1}(S(u)) & \text { if } u \in S B V(\Omega), \text { and } v \equiv 1 \\
+\infty & \text { otherwise. }
\end{array}\right.
$$

In the context of fracture, the displacement field $u$ is typically constrained by boundary values, say $U$ on $\partial \Omega$, and the crack may go to the boundary of $\Omega$. Thus we should impose that

$$
u=U \text { on } \partial \Omega \backslash S(u),
$$


$u$ being considered as an element of $S B V\left(\mathbb{R}^{2}\right)$ such that $u \equiv U$ on $\mathbb{R}^{2} \backslash \bar{\Omega}$ with $U$ defined on $\mathbb{R}^{2}$ (say $U \in H^{1}\left(\mathbb{R}^{2}\right)$ ). The relevant literature speaks of a hard device in this situation. In any case, the above quoted $\Gamma$-convergence result still applies in the current setting, so that $\mathcal{F}$, trivially extended to some $\Omega^{\prime} \supset \bar{\Omega}$, can be variationally approximated by $\mathcal{E}_{\varepsilon}$, a close variant of $A T_{\varepsilon}$ defined as

$$
\mathcal{E}_{\varepsilon}(u, v):=\int_{\Omega^{\prime}}\left(\left(\eta_{\varepsilon}+v^{2}\right)|\nabla u|^{2}+\varepsilon|\nabla v|^{2}+\frac{(1-v)^{2}}{\varepsilon}\right) \mathrm{d} x,
$$

with $(u, v) \in H^{1}\left(\Omega^{\prime}\right) \times H^{1}\left(\Omega^{\prime}\right)$ and $u \equiv U$ on $\Omega^{\prime} \backslash \bar{\Omega}$.

Remark 1.1. The extension to a larger domain $\Omega^{\prime}$ permits the introduction of boundary jumps (boundary cracks) without modification of the resulting surface energy. In the remainder of the study, we prefer to restrict the functional to $\Omega$, while imposing that the admissible fields $u$ belong to $S B V\left(\mathbb{R}^{2}\right)$ with $u=U$ on $\mathbb{R}^{2} \backslash \bar{\Omega}$. In that case, the correct surface energy for the $\Gamma\left(\mathcal{B}(\Omega) \times \mathcal{B}(\Omega)\right.$ )-limit of $\mathcal{E}_{\varepsilon}$ is

$$
2 \mathcal{H}^{1}(S(u) \cap \Omega)+\mathcal{H}^{1}(S(u) \cap \partial \Omega) .
$$

Although the functional $\mathcal{E}_{\varepsilon}$ is immediately seen to admit minimizers at fixed $\varepsilon$, those are not so easily determined computationally because $\mathcal{E}_{\varepsilon}$ is not convex in its two arguments, but only separately in each of them. This is a cause of major difficulties, as explained in [6]. The most expedient computational algorithm consists in performing alternate minimization in each variable at fixed $\varepsilon$. According to [6], that algorithm asymptotically converges to a critical point $\left(u_{\varepsilon}, v_{\varepsilon}\right)$ of $\mathcal{E}_{\varepsilon}$. Thus, algorithmically, we should investigate the limit behavior of critical pairs $\left(u_{\varepsilon}, v_{\varepsilon}\right)$ for $\mathcal{E}_{\varepsilon}$. Note that, at the expense of starting the alternate minimization with the same profile, say $v_{\varepsilon}=1, u_{\varepsilon}=U$, we can easily enforce the additional assumption that

$$
\mathcal{E}_{\varepsilon}\left(u_{\varepsilon}, v_{\varepsilon}\right) \leq \mathcal{C}<\infty,
$$

for some $\varepsilon$-independent positive constant $\mathcal{C}$.

Critical points of $\mathcal{E}_{\varepsilon}$ are not necessarily minimizers of $\mathcal{E}_{\varepsilon}$, and it is not so clear that they will converge toward even a critical point of $\mathcal{F}$. We recall, see [4], Chapter 7, that a critical point of $\mathcal{F}$ is a couple $(u, v)$ such that $\mathcal{F}$ remains stationary under admissible inner variations, i.e.,

$$
\left.\frac{\mathrm{d} \mathcal{F}\left(u \circ(i d+t \phi)^{-1}, 1\right)}{\mathrm{d} t}\right|_{t=0}=0, \text { with } \phi \in \mathcal{C}_{0}^{\infty}\left(\Omega ; \mathbb{R}^{2}\right) .
$$

If they do, then the Ambrosio-Tortorelli approximation scheme will prove even more fruitful, because fracture evolutions are more likely to be paths along critical (or maybe meta-stable) points for $\mathcal{F}$ than those along global minimizers of $\mathcal{F}$, and the result would provide a theoretical, as well as a numerical tool for extending the variational theory of brittle fracture to a more realistic setting.

Unfortunately, criticality is not easily reconciled with variational convergence. Successful attempts have been made in other settings such as that of the Allen-Cahn functional in phase transitions, see $[11,16,17]$, or that of the Ginzburg-Landau functional in superconductivity, see [5,15], but, to our knowledge, nothing of the kind has been investigated in the framework of image segmentation via the Mumford-Shah functional.

This study is a first step in that direction. It investigates the one-dimensional case. Of course, the onedimensional setting is of limited interest from the standpoint of applications to fracture, because one-dimensional fracture is primarily a textbook problem, except maybe when used in trusses. It is of marginal interest within the context of image segmentation, i.e. for $A T_{\varepsilon}$ and $M S$, although it may prove relevant for the de-blurring of bar codes [18]. Pursuing a similar analysis in a higher dimensional setting is quite a challenge for the time being. Among the many obstacles, the lack of explicit solutions for the Euler-Lagrange system associated with the criticality of the approximating fields $u_{\varepsilon}, v_{\varepsilon}$ for the Ambrosio-Tortorelli functional (see (2.4) below) makes the jump profile for $v_{\varepsilon}$ less evident than in the Allen-Cahn, or Ginzburg-Landau settings. But, the knowledge of an explicit optimal profile in those settings is a precious ingredient in the analysis of critical points. 
In the next section, the one-dimensional functional is introduced and the three main results are stated: the convergence of critical points of $\mathcal{E}_{\varepsilon}$ to specific critical points of $\mathcal{F}$ (see Thm. 2.2); conversely, any specific critical point of $\mathcal{F}$ as described in Theorem 2.2 is actually a limit of critical points of $\mathcal{E}_{\varepsilon}$ (see Thm. 2.4); finally, the convergence of the various terms in the energy $\mathcal{E}_{\varepsilon}$ to their $\mathcal{F}$-analogues (see Thm. 2.5). The reader will note that, thanks to Theorem 2.2, the Ambrosio-Tortorelli approximation acts as a selection mechanism for the Mumford-Shah functional, in that only critical points with jumps that are symmetrically located on the interval of study can be obtained through that approximation and that, thanks to Theorem 2.4, all of those are actually attained as limits of critical points of $\mathcal{E}_{\varepsilon}$. Also, Theorem 2.5 demonstrates that, generically, the AmbrosioTortorelli energy evaluated at one of its critical points converges to the Mumford-Shah energy, evaluated at the limit of that (sequence of) critical point(s). Section 3 establishes some general a priori estimates, and most notably bounds the discrepancy (see (3.3)), a pivotal quantity in the study of critical points because of its link to the energy momentum tensor (see e.g. $[5,14]$ ). Section 4 is devoted to the proof of the first theorem; Section 5 carries out that of the second theorem, while Section 6 details that of the third theorem.

\section{Statement of the Results}

Throughout, $\mathcal{C}$ stands for a generic positive constant (so that e.g. $\mathcal{C}=2 \mathcal{C}$ ) and $L$ is the length of the interval under consideration.

For $\varepsilon>0$, we consider the following $\varepsilon$-indexed one-dimensional Ambrosio-Tortorelli type functional (see (1.3)):

$$
\mathcal{E}_{\varepsilon}(u, v)=\int_{0}^{L}\left(\left(\eta_{\varepsilon}+v^{2}\right)\left(u^{\prime}\right)^{2}+\varepsilon\left(v^{\prime}\right)^{2}+\frac{(1-v)^{2}}{\varepsilon}\right) \mathrm{d} x .
$$

In $(2.1), \eta_{\varepsilon}$ is a positive number, and $\left(u_{\varepsilon}, v_{\varepsilon}\right)$ belongs to the space $Y_{\varepsilon}$ defined by

$$
Y_{\varepsilon}:=\left\{u, v \in H^{1}(0, L), u(0)=0, u(L)=a_{\varepsilon}\right\}
$$

with $a_{\varepsilon}>0$. Note that these boundary constraints are not really restrictive in one dimension (up to translation of $u$ ).

We assume that, as $\varepsilon \searrow 0$,

$$
a_{\varepsilon} \rightarrow a>0 ; \quad \eta_{\varepsilon} / \varepsilon \rightarrow 0 \text {, i.e. } \eta_{\varepsilon} \ll \varepsilon .
$$

We also introduce, for $u \in S B V(\mathbb{R})$, the one dimensional Mumford-Shah functional (see (1.2) and Rem. 1.1):

$$
\mathcal{F}(u, v)=\left\{\begin{array}{cc}
\int_{0}^{L}\left(u^{\prime}\right)^{2} \mathrm{~d} x+2 \#(S(u) \cap(0, L))+\#(S(u) \cap\{0, L\}) & \text { if } v \equiv 1 \\
+\infty & \text { otherwise. }
\end{array}\right.
$$

In (2.3), $u^{\prime}$ denotes the approximate derivative of $u$, i.e. the density of the absolutely continuous part of the measure $D u$ with respect to the Lebesgue measure, while $S(u)$ denotes the jump set of $u$, defined as the complement in $\mathbb{R}$ of the set of Lebesgue points of $u$.

As explained in the introduction, we wish to impose Dirichlet type boundary conditions on the test fields. Thus, the pair $(u, v)$ should lie in $Y$ defined as

$$
Y:=\{u \in S B V(\mathbb{R}): u \equiv 0 \text { on }(-\infty, 0), u \equiv a \text { on }(L,+\infty)\} \times L^{\infty}((0, L)),
$$

so that, in particular, $S(u) \subset[0, L]$.

The spaces $Y_{\varepsilon}$ and $Y$ are endowed with the $L^{2}\left((0, L), \mathbb{R}^{2}\right)$ topology. We recall that $\mathcal{E}_{\varepsilon} \Gamma$-converges to $\mathcal{F}$ hence minimizers of $\mathcal{E}_{\varepsilon}$ converge to minimizers of $\mathcal{F}$. Those are very easy to identify: for $a<\sqrt{L}$ the only minimizer is $u \equiv a x / L$, while for $a>\sqrt{L}$ they are $u=a \chi_{(L, \infty)}$, or $u=a \chi_{(0, \infty)}$, where $\chi$ denotes the characteristic function of a set (for $a=\sqrt{L}$ all of the above are minimizers). Thus we see that fracture is indeed induced by this 
model, even for minimizers, by a boundary "tug" when $a$ large enough. Minimization favors boundary cracks because the associated surface energy has a lesser weight (1 versus 2$)$. In the context of Remark 1.1, an energy that would weigh equally $(0, L)$ and $\{0, L\}$ would produce, for $a>\sqrt{L}$, a minimizer of the form $u=a \chi_{(b, \infty)}$ for any $b \in[0, L]$. However, our results below would prove that not all of these minimizers are produced by this limit process.

As announced in the introduction, we propose to study the convergence property of critical points other than the minimizers. The critical points of the one dimensional Mumford-Shah functional are easily identified from (7.42) in [4], Chapter 7, as those pairs $(u, v)$ with $v \equiv 1$, and $u$ piecewise constant with a finite number of jumps, or $u \equiv a x / L$.

Let $\left(u_{\varepsilon}, v_{\varepsilon}\right)$ be critical points of the Ambrosio-Tortorelli functional (2.1), i.e. pairs of functions $\left(u_{\varepsilon}, v_{\varepsilon}\right) \in Y_{\varepsilon}$ that satisfy the Euler-Lagrange equations

$$
\begin{array}{r}
-\varepsilon v_{\varepsilon}^{\prime \prime}+v_{\varepsilon}\left(u_{\varepsilon}^{\prime}\right)^{2}+\frac{v_{\varepsilon}-1}{\varepsilon}=0 \\
{\left[u_{\varepsilon}^{\prime}\left(\eta_{\varepsilon}+v_{\varepsilon}^{2}\right)\right]^{\prime}=0} \\
u_{\varepsilon}(0)=0, u_{\varepsilon}(L)=a_{\varepsilon} \\
v_{\varepsilon}^{\prime}(0)=v_{\varepsilon}^{\prime}(L)=0 .
\end{array}
$$

Our main goal is to study the limit properties of $\left(u_{\varepsilon}, v_{\varepsilon}\right)$ as $\varepsilon$ goes to 0 , provided additionally that

$$
\mathcal{E}_{\varepsilon}\left(u_{\varepsilon}, v_{\varepsilon}\right) \leq \mathcal{C}<\infty .
$$

The above bound is natural from a computational standpoint, as already emphasized in the introduction.

Note that the second equation of (2.4) implies that

$$
u_{\varepsilon}^{\prime}\left(\eta_{\varepsilon}+v_{\varepsilon}^{2}\right)=c_{\varepsilon},
$$

for some constant $c_{\varepsilon}$. It follows that $u_{\varepsilon}^{\prime}$ has a constant sign. The Dirichlet boundary conditions on $u_{\varepsilon}$ imply that $c_{\varepsilon}>0$ and thus that

$$
u_{\varepsilon} \nearrow \text { from } 0 \text { to } a_{\varepsilon} .
$$

One can substitute the relation (2.6) into the first equation of (2.4), and obtain

$$
\begin{array}{r}
-\varepsilon v_{\varepsilon}^{\prime \prime}+\frac{v_{\varepsilon} c_{\varepsilon}^{2}}{\left(\eta_{\varepsilon}+v_{\varepsilon}^{2}\right)^{2}}+\frac{v_{\varepsilon}-1}{\varepsilon}=0 \\
v_{\varepsilon}^{\prime}(0)=v_{\varepsilon}^{\prime}(L)=0 .
\end{array}
$$

It is a crucially convenient feature of the one-dimensional case that the system of ODE's can be reduced to this single second-order ODE (up to the unknown parameter $c_{\varepsilon}$ though) with Neumann boundary conditions. We will use the properties, in particular symmetry properties, of solutions to this type of ODE's. However it is not our goal to completely classify the solutions to (2.8) or perform their stability analysis. Rather we focus on the $\varepsilon \rightarrow 0$ asymptotic analysis and we look to employ, as much as possible, arguments that are independent of the dimension and could be recast in dimensions higher than 1.

Remark 2.1. Equation (2.6) would still hold true if Neumann boundary conditions, namely $u_{\varepsilon}^{\prime}(0)=u_{\varepsilon}^{\prime}(L)=0$, were imposed on $u_{\varepsilon}$, in lieu of the adopted Dirichlet boundary conditions. But then, $u_{\varepsilon}^{\prime} \equiv 0, v_{\varepsilon} \equiv 1$, and the problem becomes trivial. 


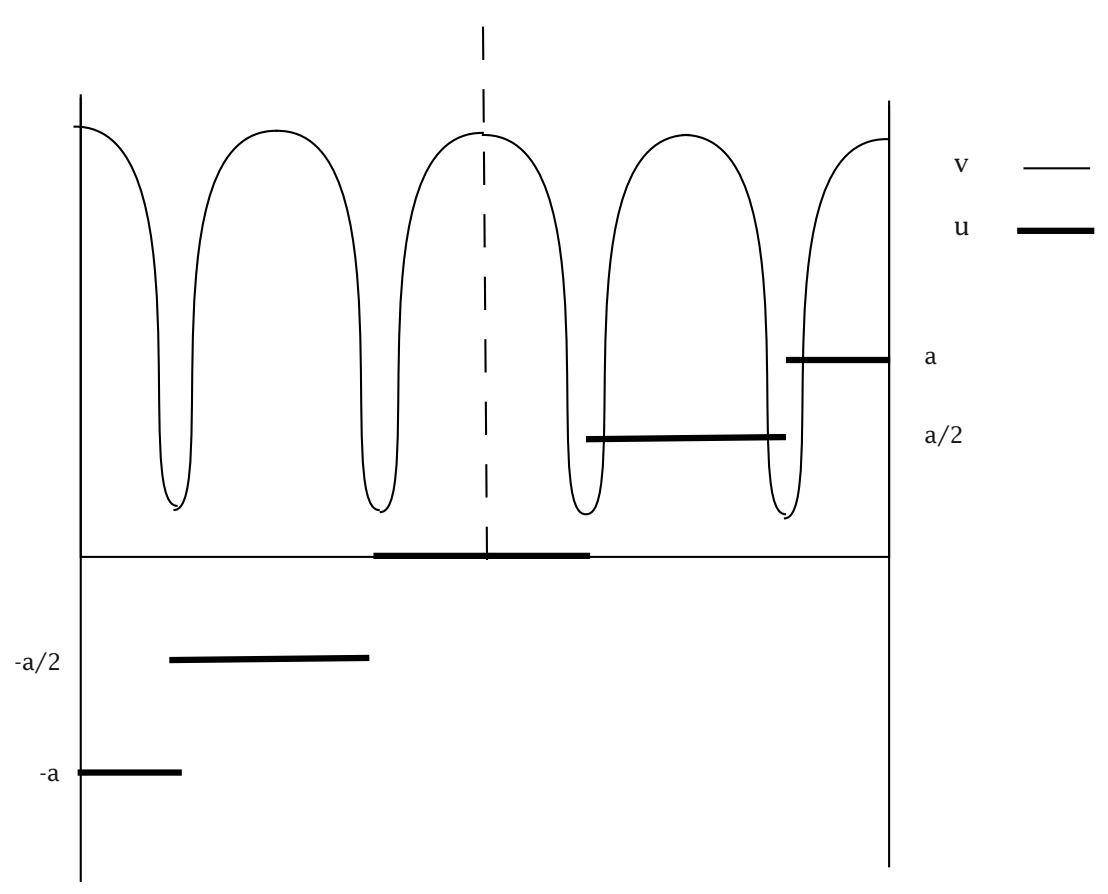

FiguRE 1. $n=4$, well case.

It is the presence of the fidelity term $\int_{0}^{L}|u-g|^{2} \mathrm{~d} x$ of image segmentation that renders the Neumann problem non-trivial. As mentioned in the introduction, our present focus is the Dirichlet case, where no fidelity term is present.

Our main result is the following. It states on the one hand the symmetry properties of the solutions to (2.4), and on the other hand the more difficult fact that $c_{\varepsilon}$ can only cluster to the two values 0 and $a / L$.

Theorem 2.2. At the possible expense of extracting a subsequence of $\varepsilon \searrow 0, c_{\varepsilon} \rightarrow c_{0}$ where $c_{0} \in\{0, a / L\}$ and $\left(u_{\varepsilon}, v_{\varepsilon}\right)$ converges to a critical point $(u, 1)$ of $\mathcal{F}$. In other words, $u_{\varepsilon}(x) \rightarrow u(x)(\in S B V(\mathbb{R}))$ and $v_{\varepsilon}(x) \rightarrow$ 1 , for a.e. $x \in(0, L)$.

If $c_{0}=a / L$, the limit critical point is $u \equiv a x / L$.

If $c_{0}=0$, there exists a fixed number $n$ such that, at the possible expense of extracting a subsequence of $\varepsilon \searrow 0$, $v_{\varepsilon}$ - extended by symmetry to $(-L, L)-i$ s a juxtaposition of $n$ identical graphs. The repeated subgraph exhibits either a strict minimum point ("well case"), for all $\varepsilon$ 's, or a strict maximum point, for all $\varepsilon$ 's ("bell case"). The limit critical point $u$ - extended by anti-parity to $(-L, L)-i$ s constant on $(-L,-L+L / n)$, with value $-a$ in the former case (see Fig. 1), or on $(-L,-L+2 L / n)$, with value $-(n-1) a / n$ in the latter case (see Fig. 2), then it jumps by a value of $2 a / n$ at the end of each interval of length $2 L / n$.

Remark 2.3. The Ambrosio-Tortorelli functional acts as a selector for the critical points of the Mumford-Shah functional, in that it asymptotically equi-distributes the possible jumps of $u$ over the interval $[0, L]$. The graph of $u$ extended by antiparity looks like a piece of a "perfect staircase": all steps have the same height and the same width.

Our next main result is a converse of the above theorem in the sense that any "perfect staircase" critical point of the Mumford-Shah functional $\mathcal{F}$ as described in Theorem 2.2 is actually a limit of critical points of the Ambrosio-Tortorelli functional $\mathcal{E}_{\varepsilon}$. 


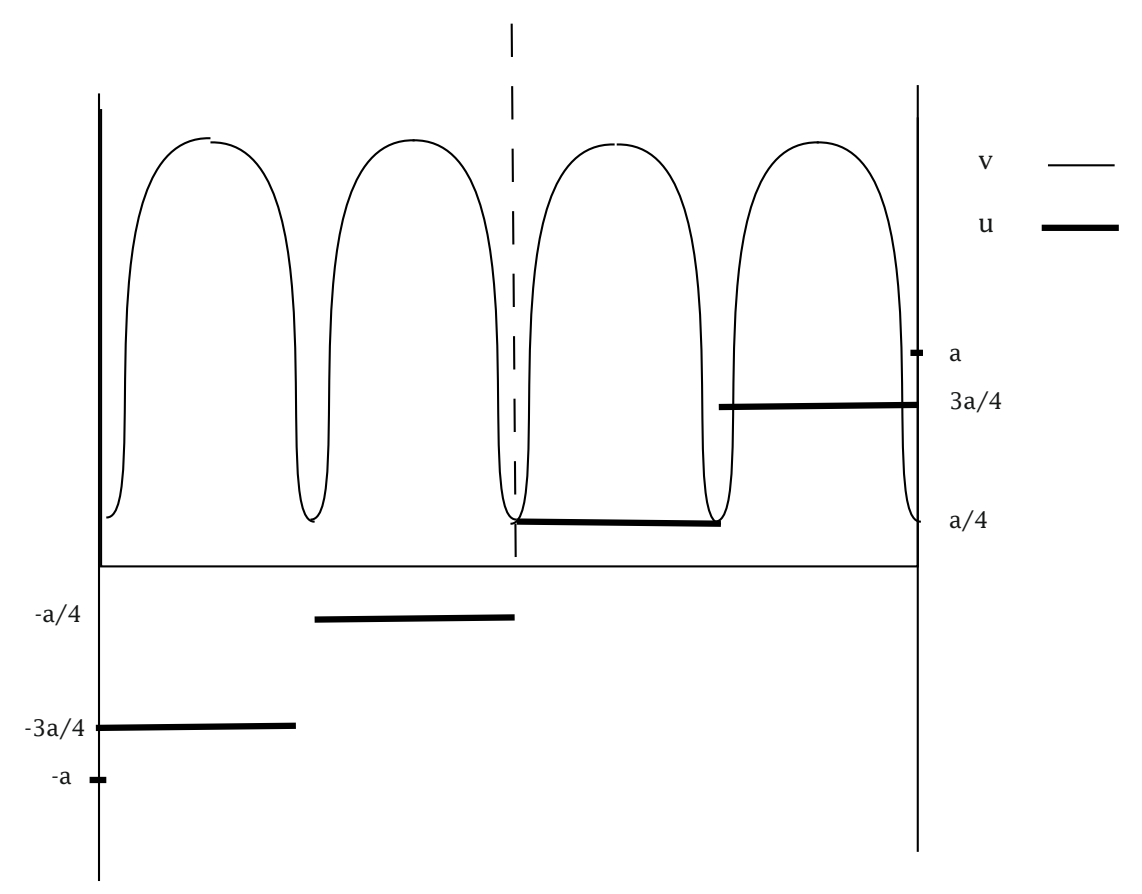

Figure 2. $n=4$, bell case.

Theorem 2.4. Let u be a "perfect staircase" function on $[0, L]$ with $n$ steps when viewed as a function on $[-L, L]$ as described in Theorem 2.2. Then, for all $\varepsilon$ sufficiently small, there exists a critical point $\left(u_{\varepsilon}, v_{\varepsilon}\right)$ (i.e. with $v_{\varepsilon}^{\prime}(0)=v_{\varepsilon}^{\prime}(L)=0$ and $\left.u_{\varepsilon}(0)=0, u_{\varepsilon}(L)=a_{\varepsilon}\right)$, of $\mathcal{E}_{\varepsilon}$ such that $v_{\varepsilon}$ has exactly $n$ isolated local maxima in $[-L, L]$ and

$$
\lim _{\varepsilon \rightarrow 0}\left\|u_{\varepsilon}-u\right\|_{L^{2}((0, L) \text { or equiv. }(-L, L))}=0 .
$$

We are also in a position to evaluate the measure limits of each of the terms entering the energy functional defined in (2.1). This is the object of the following:

Theorem 2.5. If $u_{\varepsilon} \rightarrow u$, a critical point for the Mumford-Shah functional, as given in Theorem 2.2, then

- the limit measure of $\left(\eta_{\varepsilon}+v_{\varepsilon}^{2}\right)\left(u_{\varepsilon}^{\prime}\right)^{2} \mathrm{~d} x$ is $\left(u^{\prime}\right)^{2} \mathrm{~d} x$, $u^{\prime}$, the approximate gradient of $u$, being 0 or $a / L$;

- the limit measure of $\varepsilon\left(v_{\varepsilon}^{\prime}\right)^{2} \mathrm{~d} x$, which is also that of $\left(1-v_{\varepsilon}\right)^{2} / \varepsilon \mathrm{d} x$, is a finite sum of Dirac masses which, in the case that $u$ is piecewise constant $\left(c_{0}=0\right)$, are located at the end of each step of the "perfect staircase" that represents $u$. Each of those masses has weight 1 when the mass is located inside $(0, L)$ and $1 / 2$ if it is located at $x=0, L$, if any.

Remark 2.6. In the case $c_{0}=a / L$, we expect that, for $\varepsilon$ small enough, $u_{\varepsilon}(x)=a_{\varepsilon} x / L$ and $v_{\varepsilon}(x)=L^{2} /\left(L^{2}+\right.$ $a_{\varepsilon}^{2} \varepsilon$ ), which would prove that $v_{\varepsilon}$ has no $v$-jump in the sense of Definition 3.5 below, and thus that the resulting measure limit is always $\sum_{x \in S(u) \cap(0, L)} \delta_{x}+1 / 2 \sum_{x \in S(u) \cap\{0, L\}} \delta_{x}$.

\section{Preliminary estimates}

\subsection{Classical a priori estimates}

In this section, we establish a few canonical estimates that will prove instrumental in the proof of Theorems 2.2 and 2.5. These estimates are completely standard but we include their proofs for convenience of the reader. The a priori bound (2.5) is essential in all that follows. 
First note that, from (2.5) and (2.6),

$$
\mathcal{C} \geq \mathcal{E}_{\varepsilon}\left(u_{\varepsilon}, v_{\varepsilon}\right) \geq \int_{0}^{L}\left(\eta_{\varepsilon}+v_{\varepsilon}^{2}\right)\left(u_{\varepsilon}^{\prime}\right)^{2} \mathrm{~d} x=\int_{0}^{L} c_{\varepsilon} u_{\varepsilon}^{\prime} \mathrm{d} x=a_{\varepsilon} c_{\varepsilon},
$$

so that

$$
c_{\varepsilon} \leq \frac{\mathcal{C}}{a_{\varepsilon}} .
$$

Hence, up to the possible expense of extracting a subsequence,

$$
c_{\varepsilon} \rightarrow c_{0}, \varepsilon \searrow 0
$$

The proof of Theorem 2.2 will hinge on the actual values that $c_{0}$ can take. This will be the object of Lemma 4.4 in the next section.

For now, we prove some elementary estimates on the critical points $\left(u_{\varepsilon}, v_{\varepsilon}\right)$ of $(2.1)$, which, by the way, are smooth by elliptic regularity.

A first result is a maximum principle for $v_{\varepsilon}$, namely,

\section{Lemma 3.1.}

$$
0 \leq v_{\varepsilon} \leq 1
$$

Proof. Multiplying both sides of the first equation of $(2.4)$ by $v_{\varepsilon}^{-}=\max \left(0,-v_{\varepsilon}\right)$, we get

$$
\int_{0}^{L}-\varepsilon v_{\varepsilon}^{\prime \prime} v_{\varepsilon}^{-} \mathrm{d} x+\int_{0}^{L} v_{\varepsilon}\left(u_{\varepsilon}^{\prime}\right)^{2} v_{\varepsilon}^{-} \mathrm{d} x+\int_{0}^{L} \frac{v_{\varepsilon}-1}{\varepsilon} v_{\varepsilon}^{-} \mathrm{d} x=0 .
$$

Because of the Neumann boundary conditions on $v_{\varepsilon}$, this yields

$$
\int_{0}^{L} \varepsilon v_{\varepsilon}^{\prime}\left(v_{\varepsilon}^{-}\right)^{\prime} \mathrm{d} x+\int_{0}^{L} v_{\varepsilon}\left(u_{\varepsilon}^{\prime}\right)^{2} v_{\varepsilon}^{-} \mathrm{d} x+\int_{0}^{L} \frac{v_{\varepsilon}-1}{\varepsilon} v_{\varepsilon}^{-} \mathrm{d} x=0,
$$

or still

$$
-\int_{0}^{L} \varepsilon\left(\left(v_{\varepsilon}^{-}\right)^{\prime}\right)^{2} \mathrm{~d} x-\int_{0}^{L}\left(v_{\varepsilon}^{-}\right)^{2}\left(u_{\varepsilon}^{\prime}\right)^{2} \mathrm{~d} x-\int_{0}^{L} \frac{\left(v_{\varepsilon}^{-}+1\right)}{\varepsilon} v_{\varepsilon}^{-} \mathrm{d} x=0 .
$$

Each term on the right hand side of (3.2) is nonpositive. Thus,

$$
\int_{0}^{L} \frac{\left(v_{\varepsilon}^{-}+1\right)}{\varepsilon} v_{\varepsilon}^{-} \mathrm{d} x=0
$$

hence $v_{\varepsilon}^{-} \equiv 0$.

Multiplication of the first equation of $(2.4)$ by $\left(v_{\varepsilon}-1\right)^{+}=\max \left(0, v_{\varepsilon}-1\right)$ would yield the other inequality.

Next, we establish the convergence properties of the pair $\left(u_{\varepsilon}, v_{\varepsilon}\right)$.

\section{Lemma 3.2.}

and, modulo extraction,

$$
v_{\varepsilon} \rightarrow 1, \text { strongly in } L^{2}((0, L)) \text {, }
$$

$$
\begin{gathered}
u_{\varepsilon} \rightarrow u \in B V((0, L)), \text { strongly in } L^{1}((0, L)), \\
u_{\varepsilon}^{\prime} \rightarrow c_{0}, \text { a.e. in }(0, L) .
\end{gathered}
$$

Further, $|D u|((0, L)) \leq a$ and $c_{0} \leq a / L$. 
Proof. The energy bound (2.5) immediately implies the first convergence. The monotone character (2.7) of $u_{\varepsilon}$, together with (2.2), implies that $u_{\varepsilon}$ is bounded in $B V((0, L))$. By the compactness of $B V$ in $L^{1}$ (see [9]), a subsequence of $u_{\varepsilon}$ converges in $L^{1}((0, L))$ to $u \in B V((0, L))$.

Because of the weak lower semi-continuous character of the total variation,

$$
|D u|((0, L)) \leq \liminf _{\varepsilon}\left|D u_{\varepsilon}\right|((0, L))=\liminf _{\varepsilon} a_{\varepsilon}=a,
$$

hence the bound on $|D u|((0, L))$.

By virtue of (2.6),

$$
u_{\varepsilon}^{\prime}(x) \rightarrow c_{0} \text { as } \varepsilon \rightarrow 0 \text { for a.e } x \in(0, L) .
$$

Fatou's lemma then yields the following refined bound on $c_{0}$ :

$$
c_{0} L=\int_{0}^{L} \lim _{\varepsilon \rightarrow 0} u_{\varepsilon}^{\prime} \leq \lim _{\varepsilon \rightarrow 0} \int_{0}^{L} u_{\varepsilon}^{\prime}=a .
$$

It is also standard that, in such a context, a Noether type conservation law holds, as stated in the following:

\section{Proposition 3.3.}

$$
\left\{\frac{1}{2}\left(\frac{\left(1-v_{\varepsilon}\right)^{2}}{\varepsilon}-\left(\eta_{\varepsilon}+v_{\varepsilon}^{2}\right)\left(u_{\varepsilon}^{\prime}\right)^{2}-\varepsilon\left(v_{\varepsilon}^{\prime}\right)^{2}\right)\right\}^{\prime}=0
$$

Proof. The left hand side of the previous expression also reads as

$$
\begin{aligned}
A_{\varepsilon} & :=\frac{\left(v_{\varepsilon}-1\right) v_{\varepsilon}^{\prime}}{\varepsilon}-\varepsilon v_{\varepsilon}^{\prime} v_{\varepsilon}^{\prime \prime}-v_{\varepsilon} v_{\varepsilon}^{\prime}\left(u_{\varepsilon}^{\prime}\right)^{2}-\left(v_{\varepsilon}^{2}+\eta_{\varepsilon}\right) u_{\varepsilon}^{\prime} u_{\varepsilon}^{\prime \prime} \\
& =v_{\varepsilon}^{\prime}\left(-\varepsilon v_{\varepsilon}^{\prime \prime}-v_{\varepsilon}\left(u_{\varepsilon}^{\prime}\right)^{2}+\frac{v_{\varepsilon}-1}{\varepsilon}\right)-\left(v_{\varepsilon}^{2}+\eta_{\varepsilon}\right) u_{\varepsilon}^{\prime} u_{\varepsilon}^{\prime \prime} .
\end{aligned}
$$

The first and second equation of (2.4) then imply that

$$
A_{\varepsilon}=-v_{\varepsilon}^{\prime}\left(2 v_{\varepsilon}\left(u_{\varepsilon}^{\prime}\right)^{2}\right)-\left(v_{\varepsilon}^{2}+\eta_{\varepsilon}\right) u_{\varepsilon}^{\prime} u_{\varepsilon}^{\prime \prime}=-u_{\varepsilon}^{\prime}\left[u_{\varepsilon}^{\prime \prime}\left(\eta_{\varepsilon}+v_{\varepsilon}^{2}\right)+2 v_{\varepsilon} v_{\varepsilon}^{\prime} u_{\varepsilon}^{\prime}\right]=-u_{\varepsilon}^{\prime}\left[u_{\varepsilon}^{\prime}\left(\eta_{\varepsilon}+v_{\varepsilon}^{2}\right)\right]^{\prime}=0 .
$$

An immediate consequence of the proposition above is that

$$
\frac{\left(1-v_{\varepsilon}\right)^{2}}{\varepsilon}-\left(\eta_{\varepsilon}+v_{\varepsilon}^{2}\right)\left(u_{\varepsilon}^{\prime}\right)^{2}-\varepsilon\left(v_{\varepsilon}^{\prime}\right)^{2}=d_{\varepsilon}
$$

for some constant $d_{\varepsilon}$. Furthermore, we can estimate this discrepancy constant $d_{\varepsilon}$ as follows

$$
\begin{aligned}
\left|d_{\varepsilon}\right| L=\int_{0}^{L}\left|d_{\varepsilon}\right| \mathrm{d} x & =\int_{0}^{L}\left|\frac{\left(1-v_{\varepsilon}\right)^{2}}{\varepsilon}-\left(\eta_{\varepsilon}+v_{\varepsilon}^{2}\right)\left(u_{\varepsilon}^{\prime}\right)^{2}-\varepsilon\left(v_{\varepsilon}^{\prime}\right)^{2}\right| \mathrm{d} x \\
& \leq \int_{0}^{L}\left\{\frac{\left(1-v_{\varepsilon}\right)^{2}}{\varepsilon}+\left(\eta_{\varepsilon}+v_{\varepsilon}^{2}\right)\left(u_{\varepsilon}^{\prime}\right)^{2}+\varepsilon\left(v_{\varepsilon}^{\prime}\right)^{2}\right\} \mathrm{d} x \leq \mathcal{C} .
\end{aligned}
$$

Thus,

$$
\left|d_{\varepsilon}\right| \leq \mathcal{C}
$$

This bound is key to the following gradient estimates.

Lemma 3.4. For \& small enough,

$$
\left\|u_{\varepsilon}^{\prime}\right\|_{\infty} \leq \frac{\mathcal{C}}{\left(\varepsilon \eta_{\varepsilon}\right)^{1 / 2}} \text { and }\left\|v_{\varepsilon}^{\prime}\right\|_{\infty} \leq \frac{\mathcal{C}}{\varepsilon} .
$$


Whenever $c_{0}>0$, the following refined estimates hold true:

$$
\begin{gathered}
\left\|u_{\varepsilon}^{\prime}\right\|_{\infty} \leq \frac{\mathcal{C}}{\varepsilon}, \\
v_{\varepsilon}(x) \geq \mathcal{C} \sqrt{\varepsilon}, \quad \forall x \in(0, L) .
\end{gathered}
$$

Proof. From (3.3) and (3.4), we find that

$$
\varepsilon\left(v_{\varepsilon}^{\prime}\right)^{2}=\frac{\left(1-v_{\varepsilon}\right)^{2}}{\varepsilon}-\left(\eta_{\varepsilon}+v_{\varepsilon}^{2}\right)\left(u_{\varepsilon}^{\prime}\right)^{2}-d_{\varepsilon} \leq \frac{\left(1-v_{\varepsilon}\right)^{2}}{\varepsilon}+\mathcal{C} \leq \frac{1}{\varepsilon}+\mathcal{C},
$$

from which the $L^{\infty}$-estimate of $v_{\varepsilon}^{\prime}$ follows. Moreover,

$$
c_{\varepsilon} u_{\varepsilon}^{\prime}=\left(\eta_{\varepsilon}+v_{\varepsilon}^{2}\right)\left(u_{\varepsilon}^{\prime}\right)^{2}=\frac{\left(1-v_{\varepsilon}\right)^{2}}{\varepsilon}-\varepsilon\left(v_{\varepsilon}^{\prime}\right)^{2}-d_{\varepsilon} \leq \frac{\left(1-v_{\varepsilon}\right)^{2}}{\varepsilon}+\mathcal{C} \leq \frac{1}{\varepsilon}+\mathcal{C} \leq \frac{2}{\varepsilon}
$$

from which the refined estimate on the $L^{\infty}$-norm of $u_{\varepsilon}^{\prime}$ follows if $c_{0}>0$. The lower bound for $v_{\varepsilon}$ is in turn immediate from (3.6), (2.6) and (2.2).

Finally, because $u_{\varepsilon}^{\prime}=c_{\varepsilon} /\left(\eta_{\varepsilon}+v_{\varepsilon}^{2}\right)$, we deduce from (3.6) that

$$
\left(u_{\varepsilon}^{\prime}\right)^{2} \leq \frac{2}{\varepsilon c_{\varepsilon}} \frac{c_{\varepsilon}}{\eta_{\varepsilon}+v_{\varepsilon}^{2}} \leq \frac{2}{\varepsilon \eta_{\varepsilon}}
$$

and thus obtain the first estimate on the $L^{\infty}$-norm of $u_{\varepsilon}^{\prime}$, independently of the value of $c_{0}$.

\subsection{Definition of $\boldsymbol{v}$-jump}

We start by the following remark: recalling that $u_{\varepsilon}^{\prime}=c_{\varepsilon} /\left(\eta_{\varepsilon}+v_{\varepsilon}^{2}\right)$, we may rewrite (3.3) in the form

$$
\frac{\left(1-v_{\varepsilon}\right)^{2}}{\varepsilon}-\frac{c_{\varepsilon}^{2}}{\eta_{\varepsilon}+v_{\varepsilon}^{2}}-\varepsilon\left(v_{\varepsilon}^{\prime}\right)^{2}=d_{\varepsilon}
$$

Consequently, if $x_{\varepsilon}$ is a critical point of $v_{\varepsilon}$, i.e. $v_{\varepsilon}^{\prime}\left(x_{\varepsilon}\right)=0$ and, using the fact that $v_{\varepsilon} \leq 1$, we have

$$
\left(\eta_{\varepsilon}+v_{\varepsilon}^{2}\left(x_{\varepsilon}\right)\right)\left(1-v_{\varepsilon}\left(x_{\varepsilon}\right)\right)^{2} \leq \varepsilon\left(c_{\varepsilon}^{2}+\left|d_{\varepsilon}\right|\right)
$$

It easily follows that either $v_{\varepsilon}\left(x_{\varepsilon}\right)>1-2 \sqrt{\varepsilon} \sqrt{c_{\varepsilon}^{2}+\left|d_{\varepsilon}\right|}$ or $v_{\varepsilon}\left(x_{\varepsilon}\right)<2 \sqrt{\varepsilon} \sqrt{c_{\varepsilon}^{2}+\left|d_{\varepsilon}\right|}$. Recalling that $c_{\varepsilon}$ and $\left|d_{\varepsilon}\right|$ are both bounded independently of $\varepsilon$, we can write that there exists a constant $\mathcal{C}$ such that the extremal values of $v_{\varepsilon}$ are either $>1-\mathcal{C} \sqrt{\varepsilon}$ or $<\mathcal{C} \sqrt{\varepsilon}$. Let us denote by $m_{\varepsilon}=\min _{[0, L]} v_{\varepsilon}$ and $M_{\varepsilon}=\max _{[0, L]} v_{\varepsilon}$ it follows that two cases are possible: either

$$
m_{\varepsilon}>1-\mathcal{C} \sqrt{\varepsilon}
$$

or

$$
m_{\varepsilon}<\mathcal{C} \sqrt{\varepsilon} \text { and } 1-\mathcal{C} \sqrt{\varepsilon}<M_{\varepsilon}
$$

Indeed, the case $M_{\varepsilon}<\mathcal{C} \sqrt{\varepsilon}$ would violate the energy bound (2.5).

This motivates the:

Definition 3.5. We call $x_{\varepsilon} \in[0, L]$ a v-jump if $x_{\varepsilon}$ is a critical point of $v_{\varepsilon}$ with $v_{\varepsilon}\left(x_{\varepsilon}\right) \leq \mathcal{C} \sqrt{\varepsilon}$.

In view of the above discussion, it is equivalent (for $\varepsilon$ small enough) to define $a v$-jump as a critical point of $v_{\varepsilon}$ such that $v_{\varepsilon}\left(x_{\varepsilon}\right) \leq a_{\varepsilon}$ with $a_{\varepsilon} \leq \alpha$ for any threshold value $\alpha<1$. Moreover case (3.8) happens if and only if there is no $v$-jump, and case (3.9) if and only if there is at least a $v$-jump. 
Note that the pair

is always a solution of (2.4).

$$
\left(u_{\varepsilon}, v_{\varepsilon}\right)=\left(a_{\varepsilon} x / L, L^{2} /\left(L^{2}+\varepsilon a_{\varepsilon}^{2}\right)\right)
$$

We now show that in the case (3.8), or the case of no $v$-jump, it is the only possible solution.

Lemma 3.6. If, for some $\varepsilon$ sufficiently small, $v_{\varepsilon}$ has no v-jump in $(0, L)$ then the only solution to (2.4) is $\left(u_{\varepsilon}, v_{\varepsilon}\right)=\left(a_{\varepsilon} x / L, L^{2} /\left(L^{2}+\varepsilon a_{\varepsilon}^{2}\right)\right)$.

Proof. Differentiating equation (2.6), we get $u_{\varepsilon}^{\prime \prime}\left(\eta_{\varepsilon}+v_{\varepsilon}^{2}\right)+2 v_{\varepsilon} v_{\varepsilon}^{\prime} u_{\varepsilon}^{\prime}=0$ and therefore

$$
u_{\varepsilon}^{\prime \prime}=\frac{-2 v_{\varepsilon} u_{\varepsilon}^{\prime} v_{\varepsilon}^{\prime}}{\eta_{\varepsilon}+v_{\varepsilon}^{2}}
$$

Differentiating the first equation in (2.4) gives

$$
-\varepsilon v_{\varepsilon}^{\prime \prime \prime}+v_{\varepsilon}^{\prime}\left(u_{\varepsilon}^{\prime}\right)^{2}+2 v_{\varepsilon} u_{\varepsilon}^{\prime} u_{\varepsilon}^{\prime \prime}+\frac{v_{\varepsilon}^{\prime}}{\varepsilon}=0 .
$$

Substituting (3.10) into (3.11), we find that

$$
-\varepsilon v_{\varepsilon}^{\prime \prime \prime}+v_{\varepsilon}^{\prime}\left[\left(u_{\varepsilon}^{\prime}\right)^{2}+\frac{1}{\varepsilon}-4 \frac{\left(v_{\varepsilon} u_{\varepsilon}^{\prime}\right)^{2}}{\eta_{\varepsilon}+v_{\varepsilon}^{2}}\right]=0 .
$$

With $w:=v_{\varepsilon}^{\prime}$, the above equation becomes

$$
\left\{\begin{array}{r}
-\varepsilon w^{\prime \prime}+w e_{\varepsilon}=0 \\
w(0)=0, w(L)=0
\end{array}\right.
$$

where

and substituting $(2.6)$

$$
e_{\varepsilon}=\left(u_{\varepsilon}^{\prime}\right)^{2}+\frac{1}{\varepsilon}-4 \frac{\left(v_{\varepsilon} u_{\varepsilon}^{\prime}\right)^{2}}{\eta_{\varepsilon}+v_{\varepsilon}^{2}}
$$

$$
e_{\varepsilon}=\frac{1}{\varepsilon}+\frac{c_{\varepsilon}^{2}}{\left(\eta_{\varepsilon}+v_{\varepsilon}^{2}\right)^{2}}-4 \frac{c_{\varepsilon}^{2} v_{\varepsilon}^{2}}{\left(\eta_{\varepsilon}+v_{\varepsilon}^{2}\right)^{3}}=\frac{\left(\eta_{\varepsilon}+v_{\varepsilon}^{2}\right)^{3}+\varepsilon c_{\varepsilon}^{2}\left(\eta_{\varepsilon}-3 v_{\varepsilon}^{2}\right)}{\varepsilon\left(\eta_{\varepsilon}+v_{\varepsilon}^{2}\right)^{3}} .
$$

When there is no $v$-jump then, as remarked above, $v_{\varepsilon} \geq \frac{1}{2}$ and one can easily show that then $e_{\varepsilon}>0$. Multiplying (3.12) by $w$ and after suitable integration by parts, it follows that (3.12) has a unique solution $w \equiv 0$. Thus $v_{\varepsilon}$ is a constant. Hence the result.

An obvious corollary of Lemma 3.6 is:

Remark 3.7. Whenever $c_{0}=0$, then there exists a $v$-jump for a subsequence of $\varepsilon \searrow 0$.

\section{Proof of Theorem 2.2}

\subsection{Symmetry properties}

We start by stating some relatively easy symmetry properties of the solutions to (2.4). These follow from the equation (2.8) which we recall here

$$
\left\{\begin{array}{r}
-\varepsilon v_{\varepsilon}^{\prime \prime}+\frac{v_{\varepsilon} c_{\varepsilon}^{2}}{\left(\eta_{\varepsilon}+v_{\varepsilon}^{2}\right)^{2}}+\frac{v_{\varepsilon}-1}{\varepsilon}=0 \\
v_{\varepsilon}^{\prime}(0)=v_{\varepsilon}^{\prime}(L)=0 .
\end{array}\right.
$$




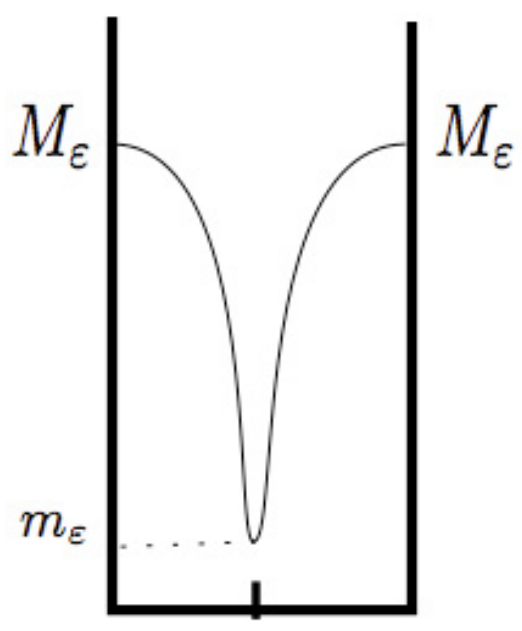

(i)

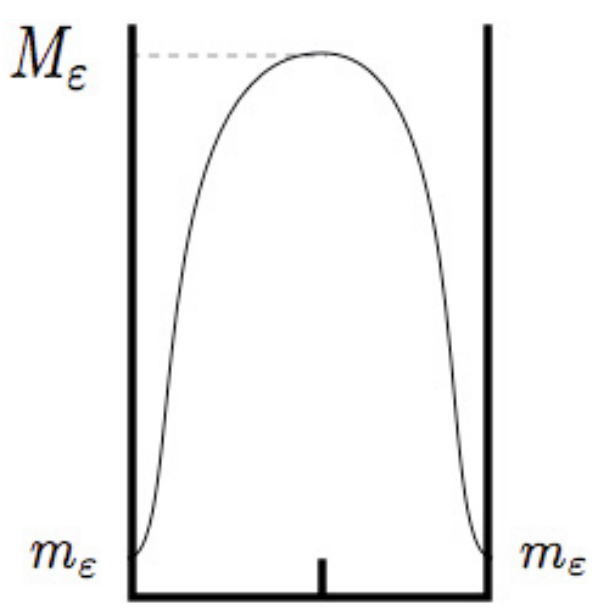

(ii)

Figure 3. (i) The well; (ii) the bell.

Observe that that equation is of the form

$$
\left\{\begin{array}{c}
v_{\varepsilon}^{\prime \prime}=f_{\varepsilon}\left(v_{\varepsilon}\right) \\
v_{\varepsilon}^{\prime}(0)=v_{\varepsilon}^{\prime}(L)=0,
\end{array}\right.
$$

with $f_{\varepsilon}$ of class $C^{2}$. Symmetry properties follow.

Lemma 4.1. The graph of $v_{\varepsilon}$ is symmetric with respect to all the vertical lines passing through its critical points, which in turn are all (including boundary points) absolute maxima or minima of $v_{\varepsilon}$.

Proof. If $x_{\varepsilon}^{c}$ is a critical point of $v_{\varepsilon}$, then we can symmetrize the graph of $v_{\varepsilon}$ through the vertical line $x=x_{\varepsilon}^{c}$. The uniqueness provided by the Cauchy-Lipschitz theorem for (4.2) imply the symmetry of the graph of $v_{\varepsilon}$ around this line. In particular the graph of $v_{\varepsilon}$ can be symmetrized with respect to $x=0$ and $v_{\varepsilon}$ can thus be extended into an even function on $[-L, L]$. Given $x_{\varepsilon}^{c}$ a critical point of $v_{\varepsilon}$, the above mentioned symmetry implies the maximality or minimality of $x_{\varepsilon}^{c}$ on $\left(-L, 2 x_{\varepsilon}^{c}+L\right)$, and ultimately, by reiteration, on $(-L, L)$.

In the sequel we will often consider this extension of $v_{\varepsilon}$ to $[-L, L]$, still denoting it $v_{\varepsilon}$.

With the same symmetry argument, we obtain the following more precise description of the graph of $v_{\varepsilon}$.

Proposition 4.2. Given $\varepsilon$, there exists an integer $n_{\varepsilon}$ such that the graph of $v_{\varepsilon}$ in $(-L, L)$ is made of $n_{\varepsilon}$ identical symmetric subgraphs. Moreover, if there is a v-jump, then each subgraph is a symmetric well with a unique interior critical point which is a v-jump, or a symmetric bell with a v-jump at each end (see Fig. 3).

It remains to show that $n_{\varepsilon}$ may be chosen independently of $\varepsilon$. To this effect, we calculate the cost of each $v$-jump for $\varepsilon$ sufficiently small. When there is a $v$-jump we are in case (3.9). Thus we can find points $\alpha<\beta$ on each subgraph satisfying say $v_{\varepsilon}(\alpha) \leq 1 / 10$ and $v_{\varepsilon}(\beta) \geq 9 / 10$. Consequently, each $v$-jump costs at least

$$
\begin{aligned}
\int_{\alpha}^{\beta}\left(\varepsilon\left(v_{\varepsilon}^{\prime}\right)^{2}+\frac{\left(1-v_{\varepsilon}\right)^{2}}{\varepsilon}\right) \mathrm{d} x & \geq \int_{\alpha}^{\beta}\left|2 v_{\varepsilon}^{\prime}\left(1-v_{\varepsilon}\right)\right| \mathrm{d} x \\
& \geq\left|\int_{\alpha}^{\beta}\left(2 v_{\varepsilon}-v_{\varepsilon}^{2}\right)^{\prime} \mathrm{d} x\right|=\left(2 v_{\varepsilon}-v_{\varepsilon}^{2}\right)(\beta)-\left(2 v_{\varepsilon}-v_{\varepsilon}^{2}\right)(\alpha) \geq 3 / 4 .
\end{aligned}
$$


Thus, because of the energy bound (2.5), $n_{\varepsilon}$ must be bounded.

So, up to possible subsequence extraction, we can consider that $n_{\varepsilon}$ is a constant $n$ for all $\varepsilon$ sufficiently small.

Remark 4.3. The uniqueness of the "well" and "bell" profiles is not clear to us at this time. If it is the case, then solutions to (2.4) would be completely determined by their number of jumps (+ boundary values).

\subsection{Characterisation of the possible limiting slopes}

We prove that $c_{\varepsilon}$ can only converge to two possible values.

Lemma 4.4. $c_{0} \in\{0, a / L\}$.

Proof. Note that, by Lemma 3.2, $c_{0} \leq a / L$. Assume by contradiction that $0<c_{0}<a / L$.

We first explain the idea of the proof. By Lemma 3.6, the difficulty in the proof can only come from the smallness of $v_{\varepsilon}$. In other words, there must be a $v$-jump. Then, let $x_{\varepsilon} \in[0, L]$ be the miniminal point of $v_{\varepsilon}$ on $[0, L]$; according to $(3.9), v_{\varepsilon}\left(x_{\varepsilon}\right) \leq \mathcal{C} \varepsilon^{1 / 2}$. Combining this with the lower bound on $v$ in Lemma 3.4, we obtain that

$$
\min _{[0, L]} v_{\varepsilon} \sim \sqrt{\varepsilon}
$$

Our proof is then based on the estimate on the size of the set $\left\{v_{\varepsilon} \leq M \sqrt{\varepsilon}\right\}$, for $M$ large enough.

Recalling that $u_{\varepsilon}^{\prime}=c_{\varepsilon} /\left(\eta_{\varepsilon}+v_{\varepsilon}^{2}\right)$, we rewrite the first equation of (2.4) as

$$
-\varepsilon v_{\varepsilon}^{\prime \prime}+\frac{v_{\varepsilon} c_{\varepsilon}^{2}}{\left(\eta_{\varepsilon}+v_{\varepsilon}^{2}\right)^{2}}+\frac{v_{\varepsilon}-1}{\varepsilon}=0 .
$$

Integrate this equation over $\left\{v_{\varepsilon} \leq M \sqrt{\varepsilon}\right\}$ to obtain

$$
\int_{\left\{v_{\varepsilon} \leq M \sqrt{\varepsilon}\right\}} \varepsilon v_{\varepsilon}^{\prime \prime} \mathrm{d} x=\int_{\left\{v_{\varepsilon} \leq M \sqrt{\varepsilon}\right\}} \frac{v_{\varepsilon} c_{\varepsilon}^{2}}{\left(\eta_{\varepsilon}+v_{\varepsilon}^{2}\right)^{2}} \mathrm{~d} x+\int_{\left\{v_{\varepsilon} \leq M \sqrt{\varepsilon}\right\}} \frac{v_{\varepsilon}-1}{\varepsilon} \mathrm{d} x .
$$

We now recall that

The number of connected components of $D_{\varepsilon}:=\left\{v_{\varepsilon} \leq M \sqrt{\varepsilon}\right\}$ is bounded by a constant $\mathcal{C}$.

Indeed the study of the previous subsection implies that the number of connected components of $D_{\varepsilon}$ is precisely the number of periods of $v_{\varepsilon}$, hence that it is bounded by $n_{\varepsilon}+1 \leq \mathcal{C}$.

On each connected component $\left(a_{i}, b_{i}\right)$ of $D_{\varepsilon}$, we obtain, by virtue of the gradient bound of Lemma 3.4,

$$
\left|\int_{a_{i}}^{b_{i}} \varepsilon v_{\varepsilon}^{\prime \prime}\right|=\left|\varepsilon v_{\varepsilon}^{\prime}\left(b_{i}\right)-\varepsilon v_{\varepsilon}^{\prime}\left(a_{i}\right)\right| \leq \mathcal{C} .
$$

Then, with (4.5), the left hand side of (4.4) is bounded from above by $\mathcal{C}$. Because $c_{0}>0$, Lemmata 3.1 and 3.4 imply that $1 \geq v_{\varepsilon}(x) \geq \mathcal{C} \sqrt{\varepsilon}, \forall x \in[0, L]$. It follows that, for $\varepsilon$ sufficiently small, $v_{\varepsilon}^{2}(x) \gg \eta_{\varepsilon}, \forall x \in[0, L]$. Thus the right hand-side is bounded from below by

$$
\int_{\left\{v_{\varepsilon} \leq M \sqrt{\varepsilon}\right\}} \frac{\mathcal{C} v_{\varepsilon}}{v_{\varepsilon}^{4}} \mathrm{~d} x-\frac{\left|\left\{v_{\varepsilon} \leq M \sqrt{\varepsilon}\right\}\right|}{\varepsilon} \geq \frac{\mathcal{C}\left|\left\{v_{\varepsilon} \leq M \sqrt{\varepsilon}\right\}\right|}{M^{3} \varepsilon^{3 / 2}}-\frac{\left|\left\{v_{\varepsilon} \leq M \sqrt{\varepsilon}\right\}\right|}{\varepsilon} \geq \frac{\mathcal{C}\left|\left\{v_{\varepsilon} \leq M \sqrt{\varepsilon}\right\}\right|}{M^{3} \varepsilon^{3 / 2}} .
$$

Therefore, for $\varepsilon$ sufficiently small,

implying in turn that

$$
\mathcal{C} \geq \frac{\mathcal{C}\left|v_{\varepsilon} \leq M \sqrt{\varepsilon}\right|}{M^{3} \varepsilon^{3 / 2}}
$$

$$
\left|\left\{v_{\varepsilon} \leq M \sqrt{\varepsilon}\right\}\right| \leq \mathcal{C} M^{3} \varepsilon^{3 / 2} \text {. }
$$


Using this inequality and the refined gradient bound for $u_{\varepsilon}$ in Lemma 3.4 yields

$$
\begin{aligned}
a_{\varepsilon}=\int_{0}^{L} u_{\varepsilon}^{\prime} \mathrm{d} x & =\int_{\left\{v_{\varepsilon} \leq M \sqrt{\varepsilon}\right\}} u_{\varepsilon}^{\prime} \mathrm{d} x+\int_{\left\{M \sqrt{\varepsilon} \leq v_{\varepsilon} \leq \frac{1}{2}\right\}} u_{\varepsilon}^{\prime} \mathrm{d} x+\int_{\left\{v_{\varepsilon} \geq \frac{1}{2}\right\}} u_{\varepsilon}^{\prime} \mathrm{d} x \\
& \leq \mathcal{C} M^{3} \varepsilon^{1 / 2}+\int_{\left\{M \sqrt{\varepsilon} \leq v_{\varepsilon} \leq \frac{1}{2}\right\}} u_{\varepsilon}^{\prime} \mathrm{d} x+\int_{0}^{L} u_{\varepsilon}^{\prime} \chi_{\left\{v_{\varepsilon} \geq \frac{1}{2}\right\}} \mathrm{d} x=: \mathcal{C} M^{3} \varepsilon^{1 / 2}+J_{\varepsilon}+K_{\varepsilon} .
\end{aligned}
$$

Next, we bound $J_{\varepsilon}$ and $K_{\varepsilon}$ from above.

Because $u_{\varepsilon}^{\prime}(x) \rightarrow c_{0}$ a.e. $x \in(0, L)$ and $\chi_{\left\{v_{\varepsilon} \geq \frac{1}{2}\right\}}(x) \rightarrow \chi_{(0, L)}(x)$ a.e $x \in(0, L)$, it follows that $w_{\varepsilon}(x):=$ $u_{\varepsilon}^{\prime}(x) \chi_{\left\{v_{\varepsilon} \geq \frac{1}{2}\right\}}(x) \rightarrow c_{0} \chi_{(0, L)}(x)$ a.e. $x \in(0, L)$. On the other hand, for all $x \in(0, L)$,

$$
\left|w_{\varepsilon}(x)\right|=\frac{c_{\varepsilon}}{\eta_{\varepsilon}+v_{\varepsilon}^{2}(x)} \chi_{\left\{v_{\varepsilon} \geq \frac{1}{2}\right\}}(x) \leq 4 c_{\varepsilon} \leq \mathcal{C} .
$$

Hence, by Lebesgue's dominated convergence theorem,

$$
K_{\varepsilon}=\int_{0}^{L} w_{\varepsilon} \mathrm{d} x \rightarrow \int_{0}^{L} c_{0} \chi_{(0, L)} \mathrm{d} x=c_{0} L
$$

From the energy bound (2.5), it follows that

$$
\mathcal{C} \geq \int_{0}^{L} \frac{\left(1-v_{\varepsilon}\right)^{2}}{\varepsilon} \mathrm{d} x \geq \int_{\left\{v_{\varepsilon} \leq 1 / 2\right\}} \frac{\left(1-v_{\varepsilon}\right)^{2}}{\varepsilon} \mathrm{d} x \geq \int_{\left\{v_{\varepsilon} \leq 1 / 2\right\}} \frac{1}{4 \varepsilon} \mathrm{d} x=\frac{1}{4 \varepsilon}\left|\left\{v_{\varepsilon} \leq 1 / 2\right\}\right|,
$$

yielding the estimate

$$
\left|\left\{M \sqrt{\varepsilon} \leq v_{\varepsilon} \leq 1 / 2\right\}\right| \leq\left|\left\{v_{\varepsilon} \leq 1 / 2\right\}\right| \leq \mathcal{C} \varepsilon
$$

On $\left\{M \sqrt{\varepsilon} \leq v_{\varepsilon} \leq 1 / 2\right\}$, we recover, for $\varepsilon$ small enough, the refined estimate on $u_{\varepsilon}^{\prime}$ from Lemma 3.4 , that is

$$
u_{\varepsilon}^{\prime}=\frac{c_{\varepsilon}}{\eta_{\varepsilon}+v_{\varepsilon}^{2}(x)} \leq \frac{c_{\varepsilon}}{v_{\varepsilon}^{2}} \leq \frac{c_{\varepsilon}}{M^{2} \varepsilon} \leq \frac{2 c_{0}}{M^{2} \varepsilon}
$$

Inserting inequalities (4.9) and (4.10) into the expression for $J_{\varepsilon}$ produces the following uniform upper bound:

$$
J_{\varepsilon}=\int_{\left\{M \sqrt{\varepsilon} \leq v_{\varepsilon} \leq \frac{1}{2}\right\}} u_{\varepsilon}^{\prime} \leq \frac{2 c_{0}}{M^{2} \varepsilon} \mathcal{C} \varepsilon=\frac{\mathcal{C}}{M^{2}}
$$


Coalescing (4.7), (4.8) and (4.11) and letting $\varepsilon$ tend to 0 finally leads to

$$
a \leq \frac{\mathcal{C}}{M^{2}}+c_{0} L
$$

We let $M$ tend to $\infty$ and obtain a contradiction since $a>c_{0} L$. Thus $c_{0} \in\{0, a / L\}$ as desired.

Remark 4.5. In this proof we have tried again to make minimal use of one-dimensional arguments. The only place where the symmetry of the solution is used for simplicity is in the proof of (4.5). But this can easily be avoided: we can show instead directly from (2.8) that the number of connected components of $D_{\varepsilon}$ is bounded by $\mathcal{C} \varepsilon^{-1 / 3}$ and this suffices in the proof.

\subsection{Form of $u$}

It remains for us to establish the form of the limit critical point. Note that $u_{\varepsilon}$ is also extended by reflection about 0 so that

$$
u_{\varepsilon}(-L)=-a_{\varepsilon} .
$$

Proposition 4.2 immediately implies the following quantization property for the function $u_{\varepsilon}$ :

$$
u_{\varepsilon}\left(-L+\frac{2 k L}{n}\right)=-a_{\varepsilon}+\frac{2 k a_{\varepsilon}}{n} \text { for } 0 \leq k \leq n .
$$

Indeed, recalling (2.6) and (4.12),

$$
2 a_{\varepsilon}=\int_{-L}^{L} \frac{c_{\varepsilon}}{\eta_{\varepsilon}+v_{\varepsilon}^{2}} \mathrm{~d} x=n \int_{-L}^{-L+2 L / n} \frac{c_{\varepsilon}}{\eta_{\varepsilon}+v_{\varepsilon}^{2}} \mathrm{~d} x=n\left(u_{\varepsilon}\left(-L+\frac{2 L}{n}\right)+a_{\varepsilon}\right) .
$$

Now, denote by $\bar{n}$ the number of times $v_{\varepsilon}$ reaches its minimal value $(\bar{n}=n$ in the well case and $\bar{n}=n+1$ in the bell case). Because of the quantization property (4.13), it suffices to consider the case $\bar{n}=1$ in the well case, and $\bar{n}=2$ in the bell case.

Assume first - well case - that $v_{\varepsilon}(-L)=v_{\varepsilon}(L)=M_{\varepsilon}$ and that $v_{\varepsilon}$ reaches its minimum (a $v$-jump) at $x=0$ and that 0 is the only critical point for $v_{\varepsilon}$ on $(-L, L)$.

Fix $\delta<L$. Then, for $\varepsilon$ sufficiently small, $v_{\varepsilon}$ converges to 1 uniformly on $K:=[\delta, L]$. Indeed, the closed set $A_{\varepsilon}:=\left\{x \in(-L, L) \mid v_{\varepsilon}(x) \leq 1-\varepsilon^{1 / 4}\right\}$ is centered around 0 , as immediately seen from the assumption that there is only one critical point at 0 . Further,

$$
\mathcal{C} \geq \int_{-L}^{L} \frac{\left(1-v_{\varepsilon}\right)^{2}}{\varepsilon} \geq \int_{A_{\varepsilon}} \frac{\left(\varepsilon^{1 / 4}\right)^{2}}{\varepsilon}=\frac{\left|A_{\varepsilon}\right|}{\varepsilon^{1 / 2}}
$$

hence $\operatorname{diam}\left(A_{\varepsilon}\right) \leq \mathcal{C} \varepsilon^{1 / 2}<\delta$ for $\varepsilon \leq \varepsilon_{0}$.

Consequently, $u_{\varepsilon}^{\prime}=c_{\varepsilon} /\left(\eta_{\varepsilon}+v_{\varepsilon}^{2}\right)$ converges uniformly to $c_{0}(=0)$ on $K$. Thus for any $x \in K$

$$
u_{\varepsilon}(x)=u_{\varepsilon}(L)-\int_{x}^{L} u_{\varepsilon}^{\prime}(t) \mathrm{d} t=a_{\varepsilon}-\int_{x}^{L} u_{\varepsilon}^{\prime}(t) \mathrm{d} t \longrightarrow a-c_{0}(L-x) .
$$

Using the arbitrariness of $\delta$, we conclude, since $c_{0}=0$, that $u=a$ on $(0, L]$. Similarly, we would find that $u=-a$ on $(-L, 0)$.

Assume now - bell case - that $v_{\varepsilon}(-L)=v_{\varepsilon}(L)=m_{\varepsilon}$ and that $v_{\varepsilon}$ reaches its maximum at $x=0$ and that 0 is the only critical point for $v_{\varepsilon}$ on $(-L, L)$. An argument identical to that above would demonstrate that $u_{\varepsilon}^{\prime}$ converges uniformly to 0 on $K:=[-L+\delta, L-\delta]$, for $\delta$ small. But we know that $u_{\varepsilon}(0)=0$, so that, for any $x \in(-L, L)$,

$$
u_{\varepsilon}(x)=u_{\varepsilon}(0)+\int_{0}^{x} u_{\varepsilon}^{\prime}(t) \mathrm{d} t=0+\int_{0}^{x} u_{\varepsilon}^{\prime}(t) \mathrm{d} t \longrightarrow c_{0} x .
$$

Using the arbitrariness of $\delta$, we conclude, since $c_{0}=0$, that $u=0$ on $(-L, L)$. 
Remark 4.6. Note that all results of this subsection hold true in the case $c_{0} \neq 0$, in particular (4.14) and (4.15), provided that $v_{\varepsilon}$ admits a $v$-jump.

Finally we examine the case $c_{0}=a / L$. If there is no $v$-jump then in view of Lemma 3.6, the result is that announced in Theorem 2.2. If there is a $v$-jump, then according to Remark 4.6, all results of the previous case hold true and, in particular (4.14) and (4.15). Hence the result upon replacing $c_{0}$ by $a / L$.

\section{Proof of Theorem 2.4}

In this section, we prove Theorem 2.4. To this end, we will use the $\Gamma$-convergence of the Ambrosio-Tortorelli functional $\mathcal{E}_{\varepsilon}$ to the Mumford-Shah functional $\mathcal{F}$ together with the equi-distribution of the $v$-jumps of any critical point $\left(u_{\varepsilon}, v_{\varepsilon}\right)$ of $\mathcal{E}_{\varepsilon}$.

Suppose now that $u$ is a piecewise constant function with $n$ steps in $[0, L]$ as described in Theorem 2.2. Because our method is general enough to handle all possible configurations of $u$, we can assume from now on that, with $N:=n-1, u \equiv 0$ on $(0, L / 2 N)$, jumps by $a / N$ at $L / 2 N$, and then jumps by a value of $a / N$ at the end of each interval of length $L / N$. We will construct critical points of $\mathcal{E}_{\varepsilon}$ - points that satisfy the Euler-Lagrange equations (2.4) - that converge to $(u, 1)$.

Before going into the details of the proof, we briefly explain the ideas. Suppose we have found $\left(u_{\varepsilon}, v_{\varepsilon}\right)$. Then, from Theorem 2.2, we know that $v_{\varepsilon}$ has a periodic structure with an equi-distribution of $v$-jumps. Furthermore, the function $u_{\varepsilon}$ enjoys a quantization property: its values at $v$-jumps are explicit, depending only on $a_{\varepsilon}, L$ and the number of jumps. By our assumption on the structure of $u$, the graph of $v_{\varepsilon}$ is expected to consist of $N$ symmetric wells on $[0, L]$. Then, we have $u_{\varepsilon}(0)=0$ and $u_{\varepsilon}(k L / 2 N)=k a_{\varepsilon} / 2 N$ for $0 \leq k \leq 2 N$. Furthermore, the graph of $v_{\varepsilon}$ on $[0, L / 2 N]$ is a half-well with a sink at $x=L / 2 N$. This sink is clearly a $v$-jump. If we are able to construct a critical point $\left(u_{\varepsilon}, v_{\varepsilon}\right)$ of $\mathcal{E}_{\varepsilon}$ on $[0, L / 2 N]$ having one $v$-jump with $u_{\varepsilon}(0)=0$ and $u_{\varepsilon}(L / 2 N)=a_{\varepsilon} / 2 N$, then we can glue appropriately identical pieces of this critical point to produce a critical point of $\mathcal{E}_{\varepsilon}$ on $[0, L]$, still denoted by $\left(u_{\varepsilon}, v_{\varepsilon}\right)$, such that $u_{\varepsilon}(0)=0, u_{\varepsilon}(L)=a_{\varepsilon}$ and also that $v_{\varepsilon}$ has $N v$-jumps and $n=N+1$ local maxima. The gluing process is always possible because the first equation of (2.4) is satisfied on each interval of length $L / 2 N$ and also, since $v_{\varepsilon}^{\prime}=0$, at the gluing points. We can thus assume without loss of generality that $N=1$ and that the $v$-jump is at $x=L$. In this case, for $\varepsilon$ small, the graph of the function $v_{\varepsilon}$, if it exists, is a half-well and $u=0$ in $[0, L)$ with $u(L)=a$. We now investigate the details.

The sought functions $v_{\varepsilon}$ clearly belong to

$$
\mathcal{U}:=\left\{v \in H^{1}(0, L): \mu \leq v(0) \leq 2-\mu ; v(L) \leq \alpha\right\}
$$

In the above definition, $0<\alpha<\mu<1$, both are independent of $\varepsilon$. Further conditions on $\mu$ and $\alpha$ will be added later on whenever necessary.

The heuristic argument above suggests that we seek local minimizers $\left(u_{\varepsilon}, v_{\varepsilon}\right)$ in the following set

$$
B_{\varepsilon}:=\left\{(u, v) \in\left(H^{1}(0, L)\right)^{2}: u(0)=0, u(L)=a_{\varepsilon}, v \in \mathcal{U}\right\} .
$$

We use the following notation:

$$
\mathcal{F}_{\varepsilon}(v, r, s):=\int_{r}^{s}\left(\varepsilon\left(v^{\prime}\right)^{2}+\frac{(1-v)^{2}}{\varepsilon}\right) \mathrm{d} x
$$

for $0 \leq r \leq s \leq L$ and note that, for $f(x)=x-x^{2} / 2$, then, for all $v \in H^{1}(0, L)$ and for all $0 \leq x_{1} \leq x_{2} \leq x_{3} \leq L$,

$$
\mathcal{F}_{\varepsilon}\left(v, x_{1}, x_{3}\right) \geq 2\left|f\left(v\left(x_{1}\right)\right)+f\left(v\left(x_{3}\right)\right)-2 f\left(v\left(x_{2}\right)\right)\right| .
$$


Indeed, assume with no loss of generality that $v \in C^{1}(0, L)$. Then, by Cauchy's inequality,

$$
\begin{aligned}
\mathcal{F}_{\varepsilon}\left(v, x_{1}, x_{3}\right)=\int_{x_{1}}^{x_{3}}\left(\varepsilon\left(v^{\prime}\right)^{2}+\frac{(1-v)^{2}}{\varepsilon}\right) \mathrm{d} x & \geq \int_{x_{1}}^{x_{3}} 2\left|v^{\prime}(1-v)\right| \mathrm{d} x \\
& =2 \int_{x_{1}}^{x_{2}}\left|\left(v-\frac{v^{2}}{2}\right)^{\prime}\right| \mathrm{d} x+2 \int_{x_{2}}^{x_{3}}\left|\left(v-\frac{v^{2}}{2}\right)^{\prime}\right| \mathrm{d} x \\
& \geq 2 \int_{x_{1}}^{x_{2}}\left(v-\frac{v^{2}}{2}\right)^{\prime} \mathrm{d} x+2 \int_{x_{2}}^{x_{3}}-\left(v-\frac{v^{2}}{2}\right)^{\prime} \mathrm{d} x \\
& =2\left(2 f\left(v\left(x_{2}\right)\right)-f\left(v\left(x_{1}\right)\right)-f\left(v\left(x_{3}\right)\right)\right) .
\end{aligned}
$$

Arguing similarly, we also obtain

$$
\mathcal{F}_{\varepsilon}\left(v, x_{1}, x_{3}\right) \geq 2\left(f\left(v\left(x_{1}\right)\right)+f\left(v\left(x_{3}\right)\right)-2 f\left(v\left(x_{2}\right)\right)\right)
$$

and thus (5.1) follows.

In a first step, we establish a universal lower bound for $\mathcal{E}_{\varepsilon}$ over $B_{\varepsilon}$ as well as an upper bound for the infimum of $\mathcal{E}_{\varepsilon}$ over $B_{\varepsilon}$. In fact, for all $(u, v) \in B_{\varepsilon}$, we have

$$
\mathcal{E}_{\varepsilon}(u, v) \geq \mathcal{F}_{\varepsilon}(v, 0, L) \geq 2(f(\mu)-f(\alpha)),
$$

while there exists $\left(\bar{u}_{\varepsilon}, \bar{v}_{\varepsilon}\right) \in B_{\varepsilon}$ such that, for $\varepsilon$ small,

$$
\mathcal{E}_{\varepsilon}\left(\bar{u}_{\varepsilon}, \bar{v}_{\varepsilon}\right) \leq 1+o(1) .
$$

To see (5.2), let $(u, v) \in B_{\varepsilon}$. Then, using (5.1) with $x_{1}=x_{2}=0$ and $x_{3}=L$, we get

$$
\mathcal{F}_{\varepsilon}(v, 0, L) \geq 2(f(v(0))-f(v(L))) \geq 2\left(\min _{x \in[\mu, 2-\mu]} f(x)-\max _{x \leq \alpha} f(x)\right)=2(f(\mu)-f(\alpha)) .
$$

Assertion (5.3) is derived upon constructing a sequence $\left(\bar{u}_{\varepsilon}, \bar{v}_{\varepsilon}\right)$ which is very similar to that used in the proof of the $\Gamma$-limsup of the Ambrosio-Tortorelli functional in [3]. We omit the details.

The direct method of the calculus of variations immediately implies that the minimum of $\mathcal{E}_{\varepsilon}$ over $B_{\varepsilon}$ is achieved at, say $\left(u_{\varepsilon}, v_{\varepsilon}\right)$. By (5.3),

$$
\mathcal{E}_{\varepsilon}\left(u_{\varepsilon}, v_{\varepsilon}\right)=\min _{(u, v) \in B_{\varepsilon}} \mathcal{E}_{\varepsilon}(u, v) \leq 1+o(1) .
$$

For a suitable choice of $(\mu, \alpha)$, a minimizer $\left(u_{\varepsilon}, v_{\varepsilon}\right)$ of $\mathcal{E}_{\varepsilon}$ over $B_{\varepsilon}$ is actually a critical point of $\mathcal{E}_{\varepsilon}$ for $\varepsilon$ sufficiently small. This is the object of the following:

Lemma 5.1. There exists $(\mu, \alpha)$, independent of $\varepsilon$, such that if $\left(u_{\varepsilon}, v_{\varepsilon}\right)$ is a minimizer of $\mathcal{E}_{\varepsilon}$ over $B_{\varepsilon}$ then it is a critical point of $\mathcal{E}_{\varepsilon}$ if $\varepsilon$ is small enough.

Proof. To prove the criticality of $\left(u_{\varepsilon}, v_{\varepsilon}\right)$, it suffices to prove the following inequalities with a suitable choice of $(\mu, \alpha)$ :

(i) $\mu<v_{\varepsilon}(0)<2-\mu$; and

(ii) $v_{\varepsilon}(L)<\alpha$.

Indeed, then $\left(u_{\varepsilon}, v_{\varepsilon}\right)$ is not on the boundary of $B_{\varepsilon}$ but rather in its interior, hence it is a local minimizer of $\mathcal{E}_{\varepsilon}$ and thus a critical point.

Our proof is by contradiction. We assume that there exists a sequence $\varepsilon_{j} \rightarrow 0$ such that either (i), or (ii) is not satisfied. Then, for each $j$, we have at least an equality in either (i), or (ii). However, through relabeling, we can assume that either (i), or (ii) is never satisfied. 
Suppose that, for all $\varepsilon$, either $v_{\varepsilon}(0)=\mu$, or $v_{\varepsilon}(0)=2-\mu$. It is enough to consider the first case because $f(\mu)=f(2-\mu)$ and only the value of $f(v(0))$ enters the proof.

We introduce the following special points $x_{\varepsilon}$. From (5.4), we know that

$$
\int_{L / 4}^{3 L / 4} \frac{\left(1-v_{\varepsilon}\right)^{2}}{\varepsilon} \mathrm{d} x \leq \mathcal{E}_{\varepsilon}\left(u_{\varepsilon}, v_{\varepsilon}\right) \leq 1+o(1) .
$$

Thus, we can find points $x_{\varepsilon} \in(L / 4,3 L / 4)$ such that $v_{\varepsilon}\left(x_{\varepsilon}\right) \rightarrow 1$ as $\varepsilon \rightarrow 0$. Then, using (5.1),

$$
\begin{aligned}
\mathcal{F}_{\varepsilon}\left(v_{\varepsilon}, 0, L\right) \geq 2\left(2 f\left(v_{\varepsilon}\left(x_{\varepsilon}\right)\right)-f\left(v_{\varepsilon}(0)\right)-f\left(v_{\varepsilon}(L)\right)\right) & \geq 2\left(2 f\left(v_{\varepsilon}\left(x_{\varepsilon}\right)\right)-f(\mu)-f(\alpha)\right) \\
& \geq 2(1-f(\mu)-f(\alpha))-o(1) .
\end{aligned}
$$

Therefore

$$
1+o(1) \geq \mathcal{E}_{\varepsilon}\left(u_{\varepsilon}, v_{\varepsilon}\right) \geq \mathcal{F}_{\varepsilon}\left(v_{\varepsilon}, 0, L\right) \geq 2(1-f(\alpha)-f(\mu))-o(1) .
$$

Thus, if we require additionally that

$$
f(\alpha)+f(\mu)<1 / 2
$$

we reach a contradiction in (5.5).

Were (ii) not true, then $v_{\varepsilon}(L)=\alpha$. Set

$$
\alpha_{\varepsilon}^{*}=\min _{x \in[0, L]} v_{\varepsilon}(x) .
$$

Then, for all $\varepsilon$, one has $\alpha_{\varepsilon}^{*} \leq \alpha$. We first improve the lower bound for $\mathcal{F}_{\varepsilon}\left(v_{\varepsilon}, 0, L\right)$ as follows

$$
\mathcal{F}_{\varepsilon}\left(v_{\varepsilon}, 0, L\right) \geq 2\left[1 / 2+\min \left\{f(\alpha)-2 f\left(\alpha_{\varepsilon}^{*}\right), 1 / 2-2 f(\alpha)\right\}\right]-o(1) .
$$

Indeed, if $y_{\varepsilon} \in(0, L]$ is such that $v_{\varepsilon}\left(y_{\varepsilon}\right)=\alpha_{\varepsilon}^{*}$, then either $x_{\varepsilon}>y_{\varepsilon}$, or $x_{\varepsilon}<y_{\varepsilon}$. If $x_{\varepsilon} \in\left(y_{\varepsilon}, L\right]$ then, applying (5.1) with $x_{1}=y_{\varepsilon}, x_{2}=x_{\varepsilon}, x_{3}=L$, and recalling that $\alpha^{*} \leq \alpha<1$, yields

$$
\begin{aligned}
\mathcal{F}_{\varepsilon}\left(v_{\varepsilon}, 0, L\right) & \geq 2\left(2 f\left(v_{\varepsilon}\left(x_{\varepsilon}\right)\right)-f\left(v_{\varepsilon}\left(y_{\varepsilon}\right)\right)-f\left(v_{\varepsilon}(L)\right)\right) \geq 2\left(1-f\left(\alpha_{\varepsilon}^{*}\right)-f(\alpha)\right)-o(1) \\
& \geq 2(1-2 f(\alpha))-o(1) .
\end{aligned}
$$

If $x_{\varepsilon}<y_{\varepsilon} \leq L$, then, applying (5.1) with $x_{1}=x_{\varepsilon}, x_{2}=y_{\varepsilon}, x_{3}=L$ yields

$$
\begin{aligned}
\mathcal{F}_{\varepsilon}\left(v_{\varepsilon}, 0, L\right) & \geq 2\left(f\left(v_{\varepsilon}\left(x_{\varepsilon}\right)\right)+f\left(v_{\varepsilon}(L)\right)-2 f\left(v_{\varepsilon}\left(y_{\varepsilon}\right)\right)\right) \\
& \geq 2\left(1 / 2+f(\alpha)-2 f\left(\alpha_{\varepsilon}^{*}\right)\right)-o(1) .
\end{aligned}
$$

Hence (5.8).

If $f(\alpha)-2 f\left(\alpha_{\varepsilon}^{*}\right)$ is strictly positive, say,

$$
f(\alpha)-2 f\left(\alpha_{\varepsilon}^{*}\right) \geq \frac{1}{2} f(\alpha)>0
$$

then we obtain a contradiction with (5.4) since

$$
1 / 2-2 f(\alpha)>0
$$

provided $\alpha$ is small enough. Thus, the only case left is $f(\alpha)-2 f\left(\alpha_{\varepsilon}^{*}\right)<\frac{1}{2} f(\alpha)$. But this case never occurs, thanks to the following: 
Proposition 5.2. Take $\alpha_{1} \in(0, \alpha)$, independent of $\varepsilon$, such that $f(\alpha) \geq 4 f\left(\alpha_{1}\right)$. Then,

$$
\min _{x \in[0, L]} v_{\varepsilon}(x)=\alpha_{\varepsilon}^{*} \leq \alpha_{1},
$$

and thus (5.9) is always satisfied.

Proof. Assume that (5.11) is false, i.e.

$$
v_{\varepsilon}(x) \geq \alpha_{1}, \quad \forall x \in[0, L] .
$$

We show that the energy $\mathcal{E}_{\varepsilon}\left(u_{\varepsilon}, v_{\varepsilon}\right)$ is actually greater than 1 and thus reach a new contradiction. Since $\left(u_{\varepsilon}, v_{\varepsilon}\right)$ minimizes $\mathcal{E}_{\varepsilon}$ over $B_{\varepsilon},\left(u_{\varepsilon}, v_{\varepsilon}\right)$ is, at the least, a critical point of $\mathcal{E}_{\varepsilon}$ on $(0, L)$ with respect to compactly supported variations in both $v_{\varepsilon}$ and $u_{\varepsilon}$. Thus, on $(0, L)$,

$$
\begin{aligned}
-\varepsilon v_{\varepsilon}^{\prime \prime}+v_{\varepsilon}\left(u_{\varepsilon}^{\prime}\right)^{2}+\frac{v_{\varepsilon}-1}{\varepsilon} & =0 \\
{\left[u_{\varepsilon}^{\prime}\left(\eta_{\varepsilon}+v_{\varepsilon}^{2}\right)\right]^{\prime} } & =0 .
\end{aligned}
$$

The second equation of (5.13) shows that, on $(0, L)$,

$$
u_{\varepsilon}^{\prime}(x)\left(\eta_{\varepsilon}+v_{\varepsilon}^{2}(x)\right)=c_{\varepsilon} \text { a.e. } x
$$

for some constant $c_{\varepsilon}$. From (5.4), (5.14), and, since $u_{\varepsilon}$ is in particular in $W^{1,1}(0, L)$,

$$
o(1)+1 \geq \mathcal{E}_{\varepsilon}\left(u_{\varepsilon}, v_{\varepsilon}\right) \geq \int_{0}^{L}\left(\eta_{\varepsilon}+v_{\varepsilon}^{2}\right)\left(u_{\varepsilon}^{\prime}\right)^{2}=\int_{0}^{L} c_{\varepsilon} u_{\varepsilon}^{\prime}=a_{\varepsilon} c_{\varepsilon},
$$

so that

$$
c_{\varepsilon} \leq \frac{\mathcal{C}}{a_{\varepsilon}} .
$$

Hence, at the possible expense of extracting a subsequence,

$$
c_{\varepsilon} \rightarrow c_{0}, \varepsilon \searrow 0 .
$$

On the other hand, the energy bound $\mathcal{E}_{\varepsilon}\left(u_{\varepsilon}, v_{\varepsilon}\right) \leq 1+o(1)$ implies that $v_{\varepsilon}$ converges to 1 in $L^{2}(0, L)$ and thus $v_{\varepsilon}(x) \rightarrow 1$ a.e. $x \in(0, L)$. Therefore

$$
u_{\varepsilon}^{\prime}(x)=\frac{c_{\varepsilon}}{\eta_{\varepsilon}+v_{\varepsilon}^{2}(x)} \rightarrow c_{0} \text { as } \varepsilon \rightarrow 0 \text { for a.e. } x \in(0, L) .
$$

In view of (5.12) and (5.14), $u_{\varepsilon}^{\prime}$ is bounded. Therefore, by Lebesgue's dominated convergence theorem,

$$
a_{\varepsilon}=\int_{0}^{L} u_{\varepsilon}^{\prime} \mathrm{d} x \rightarrow \int_{0}^{L} c_{0} \mathrm{~d} x=c_{0} L
$$

Hence $c_{0}=a / L>0$. Now,

$$
\int_{0}^{L}\left(\eta_{\varepsilon}+v_{\varepsilon}^{2}\right)\left(u_{\varepsilon}^{\prime}\right)^{2} \mathrm{~d} x=\int_{0}^{L} c_{\varepsilon} u_{\varepsilon}^{\prime} \mathrm{d} x=c_{\varepsilon} a_{\varepsilon} \geq c_{0} a-o(1) \geq \frac{a^{2}}{L}-o(1)
$$

This, together with inequality (5.2), allows us to deduce that

$$
1+o(1) \geq \mathcal{E}_{\varepsilon}\left(u_{\varepsilon}, v_{\varepsilon}\right)=\int_{0}^{L}\left(\eta_{\varepsilon}+v^{2}\right)\left(u^{\prime}\right)^{2} \mathrm{~d} x+\mathcal{F}_{\varepsilon}\left(v_{\varepsilon}, 0, L\right) \geq \frac{a^{2}}{L}+2(f(\mu)-f(\alpha))-o(1) .
$$


This is again impossible if we initially choose

$$
\frac{a^{2}}{L}+2(f(\mu)-f(\alpha))>1
$$

Summing up and recalling (5.6), (5.10) and (5.16), any minimizer $\left(u_{\varepsilon}, v_{\varepsilon}\right)$ of $\mathcal{E}_{\varepsilon}$ over $B_{\varepsilon}$ is actually a critical point of $\mathcal{E}_{\varepsilon}$ when $\varepsilon$ is small enough, provided the following conditions on $\mu$ and $\alpha$ are satisfied

$$
0<\alpha<\mu<1, f(\mu)+f(\alpha)<1 / 2,1 / 2-2 f(\alpha)>0, \frac{a^{2}}{L}+2(f(\mu)-f(\alpha))>1
$$

The set of $(\mu, \alpha)$ meeting the above requirements is not empty.

The third and final step in the proof of Theorem 2.4 is to describe the behavior of $\left(u_{\varepsilon}, v_{\varepsilon}\right)$ as $\varepsilon \rightarrow 0$. By Lemma 5.1, we easily see that $\left(u_{\varepsilon}, v_{\varepsilon}\right)$ is a local minimizer of $\mathcal{E}_{\varepsilon}$. To complete the proof, we have to prove that

(i) $v_{\varepsilon}$ has only one $v$-jump at $x=L$; and

(ii) $u$, the limit function of $u_{\varepsilon}$ in $L^{2}(0, L)$, is identically 0 on $(0, L)$.

Let us prove (i). From the definition of $v_{\varepsilon}$, we know that $v_{\varepsilon}(L) \leq \alpha<1$. We also know from Lemma 5.1 that $\left(u_{\varepsilon}, v_{\varepsilon}\right)$ is a critical point of $\mathcal{E}_{\varepsilon}$, hence $v_{\varepsilon}^{\prime}(0)=v_{\varepsilon}^{\prime}(L)=0$. Now, we invoke the remarks of Section 3.5 to conclude that $v_{\varepsilon}$ has a $v$-jump at $x=L$. If $v_{\varepsilon}$ has another $v$-jump, either at $x=0$ or at an interior point of $(0, L)$, then, using (4.3), we find that the limit energy of $\mathcal{E}_{\varepsilon}$ is at least $2 \times 3 / 4=3 / 2$. However, from (5.4), we know that the limit energy of $\mathcal{E}_{\varepsilon}\left(u_{\varepsilon}, v_{\varepsilon}\right)$ is not greater than 1 , and thus $v_{\varepsilon}$ has only one $v$-jump at $x=L$.

It remains to establish (ii). From (i), we know that $v_{\varepsilon}$ is a half-well with the sink at $x=L$. So, from Theorem 2.2, we see that either $u \equiv 0$ or $u(x)=a x / L$ for $x \in(0, L)$. The latter case cannot happen, because if it does, then by lower semicontinuity and recalling (5.2) and the fact that $v_{\varepsilon} \rightarrow 1$ in $L^{2}(0, L)$, one finds that

$$
\begin{aligned}
1 \geq \liminf _{\varepsilon \rightarrow 0} \mathcal{E}_{\varepsilon}\left(u_{\varepsilon}, v_{\varepsilon}\right) & \geq \liminf _{\varepsilon \rightarrow 0} \int_{0}^{L}\left(\eta_{\varepsilon}+v_{\varepsilon}^{2}(x)\right)\left(u_{\varepsilon}^{\prime}(x)\right)^{2} \mathrm{~d} x+\liminf _{\varepsilon \rightarrow 0} \mathcal{F}_{\varepsilon}\left(v_{\varepsilon}, 0, L\right) \\
& \geq \int_{0}^{L}\left(u^{\prime}(x)\right)^{2} \mathrm{~d} x+2(f(\mu)-f(\alpha))=\frac{a^{2}}{L}+2(f(\mu)-f(\alpha))>1,
\end{aligned}
$$

which is impossible in view of (5.17). The proof of Theorem 2.4 is now complete.

Remark 5.3. If $N=1$ and the $v$-jump is at the boundary of $(0, L)$ then the critical points $\left(u_{\varepsilon}, v_{\varepsilon}\right)$ of $\mathcal{E}_{\varepsilon}$ constructed in Theorem 2.4 are also local minimizers of $\mathcal{E}_{\varepsilon}$. We conjecture that if $N=1$ and the $v$-jump is at $L / 2$ or if $N \geq 2$, then the critical points $\left(u_{\varepsilon}, v_{\varepsilon}\right)$ found in Theorem 2.4 are also local minimizers of $\mathcal{E}_{\varepsilon}$.

\section{Proof of Theorem 2.5}

The measure limit of $\left(\eta_{\varepsilon}+v_{\varepsilon}^{2}\right)\left(u_{\varepsilon}^{\prime}\right)^{2} \mathrm{~d} x$ is immediately computed upon remarking that, thanks to (2.6),

$$
\left(\eta_{\varepsilon}+v_{\varepsilon}^{2}\right)\left(u_{\varepsilon}^{\prime}\right)^{2}=c_{\varepsilon} u_{\varepsilon}^{\prime}
$$

so that the measure limit of $\left(\eta_{\varepsilon}+v_{\varepsilon}^{2}\right)\left(u_{\varepsilon}^{\prime}\right)^{2} \mathrm{~d} x$ is that of $c_{\varepsilon} u_{\varepsilon}^{\prime} \mathrm{d} x$. Testing with a smooth compactly supported function $\varphi$, we obtain

$$
\int_{0}^{L} c_{\varepsilon} u_{\varepsilon}^{\prime} \varphi \mathrm{d} x=-c_{\varepsilon} \int_{0}^{L} u_{\varepsilon} \varphi^{\prime} \mathrm{d} x \longrightarrow-c_{0} \int_{0}^{L} u \varphi^{\prime} \mathrm{d} x
$$

so that, upon recalling Theorem 2.2, we obtain the desired result. The reader will have not failed to note the fortuitousness of the value of $c_{0}$, i.e. 0 , when $u$ jumps; the result would be false if $u$ could jump for non-zero values of $c_{0}$ ! 
The computation of the measure limit of the other two terms is more delicate. We first establish that there is no concentration of energy for those two terms away from the jump points. We will only use the property that there is a finite $\varepsilon$-independent number $j$ of $v$-jumps on $[0, L]$, denoted by $x_{\varepsilon}^{1}, \ldots, x_{\varepsilon}^{j}$, with $x_{\varepsilon}^{k} \rightarrow x_{k}, k=1, \ldots, j$.

Lemma 6.1. For any compact subset $K \subset[0, L] \backslash \cup_{k=1, \ldots, j}\left\{x_{k}\right\}$, we have

$$
\int_{K}\left(\varepsilon\left(v_{\varepsilon}^{\prime}(x)\right)^{2}+\left(1-v_{\varepsilon}(x)\right)^{2} / \varepsilon\right) \mathrm{d} x \leq \mathcal{C}_{K} \varepsilon^{1 / 4},
$$

where $\mathcal{C}_{K}$ may depend only on $K$.

Proof. Consider $A_{\varepsilon}:=\left\{x \in[0, L]: v_{\varepsilon}(x) \leq 1-\varepsilon^{1 / 4}\right\}$. Then, from the energy bound $(2.5),\left|A_{\varepsilon}\right| \leq \mathcal{C} \sqrt{\varepsilon}$, while $\left\{x_{\varepsilon}^{1}, \ldots, x_{\varepsilon}^{j}\right\} \subset A_{\varepsilon}$. For a given compact subset $K$ of $[0, L] \backslash \cup_{k=1, \ldots, j}\left\{x_{k}\right\}$, set $\delta:=1 / 2 \min _{k=1, \ldots, j} \operatorname{dist}\left(x_{k}, K\right)$. If $\varepsilon$ is small enough, $\left\{x_{1}, \ldots, x_{j}\right\} \subset A_{\varepsilon} \subset U_{\delta}:=\cup_{k=1, \ldots, j}\left[x_{k}-\delta, x_{k}+\delta\right]$, so that, $K \cap A_{\varepsilon}=\emptyset$. Because $K \subset[0, L] \backslash U_{\delta}$, it suffices to prove that

$$
\int_{[0, L] \backslash U_{\delta}}\left(\varepsilon\left(v_{\varepsilon}^{\prime}(x)\right)^{2}+\left(1-v_{\varepsilon}(x)\right)^{2} / \varepsilon\right) \mathrm{d} x \leq \mathcal{C}_{K} \varepsilon^{1 / 4} .
$$

Let us denote $V_{\delta}=[0, L] \backslash U_{\delta}$. Multiplying both sides of the first equation of $(2.4)$ by $v_{\varepsilon}-1$ and integrating over $V_{\delta}$, we get

$$
\int_{V_{\delta}}-\varepsilon v_{\varepsilon}^{\prime \prime}(x)\left(v_{\varepsilon}(x)-1\right) \mathrm{d} x+\int_{V_{\delta}} v_{\varepsilon}(x)\left(u_{\varepsilon}^{\prime}(x)\right)^{2}\left(v_{\varepsilon}(x)-1\right) \mathrm{d} x+\int_{V_{\delta}} \frac{\left(v_{\varepsilon}(x)-1\right)^{2}}{\varepsilon} \mathrm{d} x=0 .
$$

Note that $V_{\delta}$ is a union of a finite $\varepsilon$-independent number $J(\leq j+1)$ of intervals on $[0, L]: V_{\delta}=\cup_{k=1, \ldots, J}\left[a_{\varepsilon}^{k}, b_{\varepsilon}^{k}\right]$. Now, integrating by parts the first term of (6.2), recalling (2.6) and rearranging, one obtains

$$
\begin{aligned}
\int_{V_{\delta}} \varepsilon\left(v_{\varepsilon}^{\prime}(x)\right)^{2} \mathrm{~d} x+\int_{V_{\delta}} \frac{\left(v_{\varepsilon}(x)-1\right)^{2}}{\varepsilon} \mathrm{d} x=\sum_{k=1}^{J} \varepsilon\left(v_{\varepsilon}^{\prime}\left(b_{\varepsilon}^{k}\right)\left(v_{\varepsilon}\left(b_{\varepsilon}^{k}\right)-1\right)\right. & \left.-v_{\varepsilon}^{\prime}\left(a_{\varepsilon}^{k}\right)\left(v_{\varepsilon}\left(a_{\varepsilon}^{k}\right)-1\right)\right) \\
& +\int_{V_{\delta}} \frac{c_{\varepsilon}^{2}}{\left(\eta_{\varepsilon}+v_{\varepsilon}^{2}(x)\right)^{2}} v_{\varepsilon}(x)\left(1-v_{\varepsilon}(x)\right) \mathrm{d} x .
\end{aligned}
$$

By the definitions of $A_{\varepsilon}$ and $V_{\delta}$, we have $\left|1-v_{\varepsilon}\right| \leq \varepsilon^{1 / 4}$ on $V_{\delta}$. Combining this fact with the gradient bound for $v_{\varepsilon}$ in Lemma 3.4 yields that the right hand side of $(6.3)$ is bounded from above by $\mathcal{C}_{K} \varepsilon^{1 / 4}$ for some constant $\mathcal{C}_{K}$ which may depend only on $K$. Hence the desired result stated in (6.1) follows.

Remark 6.2. The previous lemma shows that the measure limits of $\varepsilon\left(v_{\varepsilon}^{\prime}(x)\right)^{2} \mathrm{~d} x$, and of $\left(v_{\varepsilon}(x)-1\right)^{2} / \varepsilon \mathrm{d} x$ are Dirac masses concentrated at $x_{1}, \ldots, x_{j}$. We will evaluate their respective weight in the fourth and final step below.

Also note that, thanks to Lemma 3.6, those limits are immediately computed (and found to be 0 !) in the absence of $v$-jumps.

The second step consists in computing the limit $d_{0}$ of the discrepancy $d_{\varepsilon}$ defined in (3.3), which exists, at least for a well chosen subsequence, by the boundedness (3.4) of $d_{\varepsilon}$. To this effect, we prove the following:

Lemma 6.3. $d_{0}+c_{0}^{2}=0$.

Proof. Recalling (3.3) and (2.6), we obtain

$$
d_{\varepsilon}+c_{\varepsilon} u_{\varepsilon}^{\prime}(x)=\frac{\left(v_{\varepsilon}(x)-1\right)^{2}}{\varepsilon}-\varepsilon\left(v_{\varepsilon}^{\prime}(x)\right)^{2}
$$

and thus

$$
\left|d_{\varepsilon}+c_{\varepsilon} u_{\varepsilon}^{\prime}(x)\right|=\left|\frac{\left(v_{\varepsilon}(x)-1\right)^{2}}{\varepsilon}-\varepsilon\left(v_{\varepsilon}^{\prime}(x)\right)^{2}\right| .
$$


Let $A_{\varepsilon}, K$ with $|K|>0$ and $\delta$ be as in the proof of Lemma 6.1. Then, on $K$, one has $1-\varepsilon^{1 / 4} \leq v_{\varepsilon} \leq 1$. By virtue of (2.6), $u_{\varepsilon}^{\prime}$ is bounded on $K$. Upon integrating (6.5) over $K$, recalling Lemma 3.2 and letting $\varepsilon \searrow 0$, we have by Lebesgue's dominated convergence theorem

$$
|K|\left|d_{0}+c_{0}^{2}\right|=\lim _{\varepsilon \rightarrow 0} \int_{K}\left|d_{\varepsilon}+c_{\varepsilon} u_{\varepsilon}^{\prime}(x)\right| \mathrm{d} x=\lim _{\varepsilon \rightarrow 0} \int_{K}\left|\frac{\left(v_{\varepsilon}(x)-1\right)^{2}}{\varepsilon}-\varepsilon\left(v_{\varepsilon}^{\prime}(x)\right)^{2}\right| \mathrm{d} x .
$$

In view of Lemma 6.1, the last term of the above equation is 0 . Thus $d_{0}+c_{0}^{2}=0$ as claimed.

The third step consists of the following equi-partition result:

Lemma 6.4. For all $x$ in $[0, L]$,

$$
\left|v_{\varepsilon}^{\prime}(x)\right| \leq \frac{1-v_{\varepsilon}(x)}{\varepsilon}
$$

Further,

$$
\lim _{\varepsilon \rightarrow 0} \int_{0}^{L}\left|\varepsilon\left(v_{\varepsilon}^{\prime}(x)\right)^{2}-\left(v_{\varepsilon}(x)-1\right)^{2} / \varepsilon\right| \mathrm{d} x=0 .
$$

Proof. According to (6.4), the term $\left(v_{\varepsilon}(x)-1\right)^{2} / \varepsilon-\varepsilon\left(v_{\varepsilon}^{\prime}(x)\right)^{2}$ attains, for a fixed $\varepsilon$, its minimum on $[0, L]$ precisely where $u_{\varepsilon}^{\prime}=c_{\varepsilon} /\left(\eta_{\varepsilon}+v_{\varepsilon}^{2}\right)$ attains its minimum, or still, where $v_{\varepsilon}$ attains its maximum. But, at such points, $v_{\varepsilon}^{\prime}$ cancels, so that the minimum of that term is non-negative. Thus,

$$
\begin{aligned}
\int_{0}^{L}\left|\frac{\left(v_{\varepsilon}(x)-1\right)^{2}}{\varepsilon}-\varepsilon\left(v_{\varepsilon}^{\prime}(x)\right)^{2}\right| \mathrm{d} x & =\int_{0}^{L}\left(\frac{\left(v_{\varepsilon}(x)-1\right)^{2}}{\varepsilon}-\varepsilon\left(v_{\varepsilon}^{\prime}(x)\right)^{2}\right) \mathrm{d} x \\
& =\int_{0}^{L}\left(d_{\varepsilon}+c_{\varepsilon} u_{\varepsilon}^{\prime}(x)\right) \mathrm{d} x=L d_{\varepsilon}+c_{\varepsilon} a_{\varepsilon} \\
& \rightarrow L d_{0}+c_{0} a=L\left(d_{0}+c_{0}^{2}\right)=0,
\end{aligned}
$$

in view of Lemma 6.3.

The fourth and final step consists in evaluating the respective weights of the Dirac masses making up the limit of $\varepsilon\left(v_{\varepsilon}^{\prime}(x)\right)^{2} \mathrm{~d} x$ and of $\left(v_{\varepsilon}(x)-1\right)^{2} / \varepsilon \mathrm{d} x$.

To this effect, we first remark that, at the possible expense of extending $v_{\varepsilon}$ by reflection around $x=0$ and/or $x=L$, we may always compute the measure limit $\mu$ of $\left(\varepsilon\left(v_{\varepsilon}^{\prime}(x)\right)^{2}+\left(v_{\varepsilon}(x)-1\right)^{2} / \varepsilon\right) \mathrm{d} x$ over some $I \supset[0, L]$, so that $\mu(\partial I)=0$, in which case $\mu(I)=\lim _{\varepsilon} \int_{I}\left(\varepsilon\left(v_{\varepsilon}^{\prime}(x)\right)^{2}+\left(v_{\varepsilon}(x)-1\right)^{2} / \varepsilon\right) \mathrm{d} x$, while Lemma 6.4 still applies over $I$.

Note that

$$
\begin{aligned}
\int_{I}\left(\varepsilon\left(v_{\varepsilon}^{\prime}(x)\right)^{2}+\frac{\left(v_{\varepsilon}(x)-1\right)^{2}}{\varepsilon}-2\left|v_{\varepsilon}^{\prime}(x)\right|\left(1-v_{\varepsilon}(x)\right)\right) \mathrm{d} x & =\int_{I}\left(\varepsilon^{1 / 2}\left|v_{\varepsilon}^{\prime}(x)\right|-\frac{\left(1-v_{\varepsilon}(x)\right)}{\varepsilon^{1 / 2}}\right)^{2} \mathrm{~d} x \\
& \leq \int_{I}\left|\varepsilon\left(v_{\varepsilon}^{\prime}(x)\right)^{2}-\frac{\left(1-v_{\varepsilon}(x)\right)^{2}}{\varepsilon}\right| \mathrm{d} x
\end{aligned}
$$

which goes to 0 with $\varepsilon$, according to Lemma 6.4 above. Thus, the total mass of the measure limit of $\left(\varepsilon\left(v_{\varepsilon}^{\prime}(x)\right)^{2}+\right.$ $\left.\left(v_{\varepsilon}(x)-1\right)^{2} / \varepsilon\right) \mathrm{d} x$, is also that of $2\left|v_{\varepsilon}^{\prime}(x)\right|\left(1-v_{\varepsilon}(x)\right) \mathrm{d} x$. But, we know, according to Proposition 4.2, that the graph of $v_{\varepsilon}$ is symmetric around each $v$-jump, so that it suffices to compute the mass of measure limit of $2\left|v_{\varepsilon}^{\prime}(x)\right|\left(1-v_{\varepsilon}(x)\right) \mathrm{d} x$ over a half well, that is,

$$
\int_{\left\{x: v_{\varepsilon}(x) \in\left[m_{\varepsilon}, M_{\varepsilon}\right]\right\}} 2\left|v_{\varepsilon}^{\prime}(x)\right|\left(1-v_{\varepsilon}(x)\right) \mathrm{d} x,
$$


or still, since $v_{\varepsilon}^{\prime}(x)>0$ on $\left\{x: v_{\varepsilon}(x) \in\left[m_{\varepsilon}, M_{\varepsilon}\right]\right\}$,

$$
\int_{m_{\varepsilon}}^{M_{\varepsilon}} 2(1-y) \mathrm{d} y=\left(2 M_{\varepsilon}-M_{\varepsilon}^{2}\right)-\left(2 m_{\varepsilon}-m_{\varepsilon}^{2}\right) \longrightarrow 1,
$$

as $\varepsilon \searrow 0$, since $M_{\varepsilon} \nearrow 1$, while $m_{\varepsilon} \searrow 0$. Hence the measure limit $\mu$ is given by

$$
\mu=2 \sum_{\{x: x \text { is a } v \text {-jump }\}} \delta_{x} .
$$

The proof of Theorem 2.5 is now complete.

Acknowledgements. The authors are grateful to the referees for their comments and criticisms which resulted in a hopefully improved version of the original manuscript.

\section{REFERENCES}

[1] L. Ambrosio, Existence theory for a new class of variational problems. Arch. Ration. Mech. Anal. 111 (1990) $291-322$.

[2] L. Ambrosio and V.M. Tortorelli, Approximation of functionals depending on jumps by elliptic functionals via $\Gamma$-convergence. Comm. Pure Appl. Math. 43 (1990) 999-1036.

[3] L. Ambrosio and V.M. Tortorelli, On the approximation of free discontinuity problems. Boll. Un. Mat. Ital. B (7) 6 (1992) $105-123$.

[4] L. Ambrosio, N. Fusco and D. Pallara, Functions of Bounded Variation and Free Discontinuity Problems. Oxford University Press (2000).

[5] F. Bethuel, H. Brezis and F. Hélein, Ginzburg-Landau vortices, Progress in Nonlinear Differential Equations and their Applications 13. Birkhäuser Boston Inc., Boston, MA (1994).

[6] B. Bourdin, Numerical implementation of the variational formulation of brittle fracture. Interfaces Free Bound. 9 (2007) 411-430.

[7] A. Braides, $\Gamma$-convergence for Beginners, Oxford Lecture Series in Mathematics and its Applications 22. Oxford University Press (2002).

[8] E. De Giorgi, M. Carriero and A. Leaci, Existence theorem for a minimum problem with free discontinuity set. Arch. Ration. Mech. Anal. 108 (1989) 195-218.

[9] L.C. Evans and R.F. Gariepy, Measure theory and fine properties of functions. CRC Press, Boca Raton, FL (1992).

[10] G.A. Francfort and J.-J. Marigo, Revisiting brittle fracture as an energy minimization problem. J. Mech. Phys. Solids 46 (1998) 1319-1342.

[11] J.E. Hutchinson and Y. Tonegawa, Convergence of phase interfaces in the van der Waals-Cahn-Hilliard theory. Calc. Var. Partial Differential Equations 10 (2000) 49-84.

[12] L. Modica and S. Mortola, Il limite nella $\Gamma$-convergenza di una famiglia di funzionali ellittici. Boll. Un. Mat. Ital. A (5) 14 (1977) 526-529.

[13] D. Mumford and J. Shah, Optimal approximations by piecewise smooth functions and associated variational problems. Comm. Pure Appl. Math. XLII (1989) 577-685.

[14] P.J. Olver, Applications of Lie groups to differential equations, Graduate Texts in Mathematics 107. Springer-Verlag, New York (1986).

[15] E. Sandier and S. Serfaty, Vortices in the magnetic Ginzburg-Landau model, Progress in Nonlinear Differential Equations and their Applications 70. Birkhäuser Boston Inc., Boston, MA (2007).

[16] Y. Tonegawa, Phase field model with a variable chemical potential. Proc. Roy. Soc. Edinburgh Sect. A 132 (2002) $993-1019$.

[17] Y. Tonegawa, A diffused interface whose chemical potential lies in a Sobolev space. Ann. Sc. Norm. Super. Pisa Cl. Sci. (5) 4 (2005) 487-510.

[18] T. Wittman, Lost in the supermarket: decoding blurry barcodes. SIAM News $\mathbf{3 7}$ September (2004). 